
\title{
R Reserach S Suare \\ Liver kinase B1 rs9282860 polymorphism and risk for multiple sclerosis in White and Black Americans
}

\author{
Anne I Boullerne \\ University of Illinois at Chicago \\ Mitchell T Wallin \\ VA Multiple Sclerosis Center of Excellence - East \\ William J Culpepper \\ VA Multiple Sclerosis Center of Excellence - East \\ Heidi Maloni \\ VA Multiple Sclerosis Center of Excellence - East \\ Elizabeth A. Boots \\ Rush University Medical Center

\section{Dagmar M Sweeney} \\ University of Illinois at Chicago \\ Douglas L Feinstein ( $\nabla$ dlfeins@uic.edu ) \\ Jesse Brown VA Medical Center
}

\section{Research Article}

Keywords: STK11, African American, multiple sclerosis, SNP, military Veterans, race and ethnicity, gender, progressive form

Posted Date: February 22nd, 2021

DOI: https://doi.org/10.21203/rs.3.rs-264811/v1

License: (c) (i) This work is licensed under a Creative Commons Attribution 4.0 International License.

Read Full License 
Liver kinase B1 rs9282860 polymorphism and risk for multiple sclerosis in White and Black Americans

Anne I Boullerne ${ }^{1}$, Mitchell T Wallinn ${ }^{2,3,4}$, William J Culpepper ${ }^{2,4}$, Heidi Maloni ${ }^{2}$, Elizabeth A. Boots ${ }^{5,6}$, Dagmar M Sweeney ${ }^{7}$, Douglas L Feinstein ${ }^{1,8}$

${ }^{1}$ Department of Anesthesiology, University Illinois, Chicago, IL

2 VA Multiple Sclerosis Center of Excellence-East, Washington, DC

${ }^{3}$ George Washington University School of Medicine in Washington, DC

${ }^{4}$ Department of Neurology, University Maryland, Baltimore, MD

${ }^{5}$ Department of Psychology, University Illinois, Chicago, IL

${ }^{6}$ Rush Alzheimer's Disease Center, Rush University Medical Center, Chicago, IL

${ }^{7}$ Research Resources Center, University Illinois, Chicago, IL

8 Jesse Brown VA Medical Center, Chicago, IL

Correspondence:

$835 \mathrm{~S}$ Wolcott St

MC513/E720

Chicago, IL, 60614

\section{Keywords}

STK11; African American; multiple sclerosis; SNP; military Veterans; race and ethnicity; gender; progressive form.

\section{Words: 4,500 Tables: 4 Figures: 7 Supplemental Tables: 1}

\section{Support}

This work was supported by Merit grant I01BX002625 (DLF) and Research Career Scientist award IK6BX004852 (DLF) from the Department of Veterans Affairs; grant HC-1508-05693 from the National MS Society (MTW); and grant SI-2001-35701 from the National MS Society which supports the UCSF MS biorepository. The VALOMS study was supported by the Veterans Affairs Multiple Sclerosis Center of Excellence.

\section{Acknowledgments}

We thank Walter Royal, III (Morehouse School of Medicine, Georgia) and all VALOMS Investigators; David Gwynne and Sara Loud (Accelerated Cure Project), and Jorge Oksenberg and Stacy Caillier (UCSF) for assistance providing samples; Stefan Green (UIC) for assistance with Taqman assays; and Mark Maienschein-Cline (UIC) for assistance with statistical analyses. 


\begin{abstract}
We previously reported that a single nucleotide polymorphism (SNP) rs9282860 in serine threonine kinase 11 (STK11) gene which codes for liver kinase B1 (LKB1) has higher prevalence in White relapsing-remitting multiple sclerosis (RRMS) patients than controls. To determine if this SNP is a risk factor for MS in other populations, we assessed its prevalence in samples collected from African American (AA) persons with MS (PwMS) and controls at multiple Veterans Affairs (VA) Medical Centers and from a network of academic MS centers. There were no significant differences in average age at first symptom onset, diagnosis, disease duration, or disease severity between RRMS patients recruited from VAMCs versus non-VAMCs. The SNP was more prevalent in AA than White PwMS, however only in secondary progressive MS (SPMS) patients was that difference statistically significant. AA SPMS patients had higher STK11 SNP prevalence than non-MS controls; and in that cohort the SNP was associated with older age at symptom onset and at diagnosis. The results suggest that the STK11 SNP represents a risk factor for SPMS in AA patients, and is associated with an older age of symptom onset and diagnosis.
\end{abstract}




\section{Introduction}

Genetic studies have identified over 200 single nucleotide polymorphisms (SNPs) associated with an increased risk of multiple sclerosis (MS) [1-5]; however, the majority of studies were done in persons of European ancestry. In contrast, few studies have examined the association of genetic variants with MS in African American (AA) populations, and of those most examined polymorphisms within major histocompatibility locus human leukocyte antigen (HLA) alleles [6-10]. Outside the MHC region, several MS variants were shown to replicate in AA populations, although some studies [11,12] reported low replication pointing to significant heterogeneity amongst AA populations. Differences in genetic risk factors for MS could contribute to differences in MS incidence, prevalence or progression between AA and White populations [13-16].

We previously reported that a single nucleotide polymorphism (SNP) in the gene STK11 (serinethreonine kinase 11), which encodes LKB1 (liver kinase B1), is a risk factor in White RR (relapsingremitting) patients with MS (PwMS), with an odds ratio (OR) of 1.66 in females [17]. LKB1 is a ubiquitously expressed kinase, which phosphorylates and activates multiple downstream kinases [18] regulating cell functions including metabolism, migration, and proliferation. LKB1 is a metabolic sensor to help maintain ATP levels during periods of intense activity and stress [19]. LKB1 also regulates proinflammatory cytokine production from T-cells [20]; and proliferation of T-regulatory cells [21]. Conditional depletion of LKB1 from Schwann cells revealed a role in peripheral myelination [22-24]; while conditional depletion from astrocytes exacerbated disease progression in a mouse model of MS, associated with increased neuroinflammation and damage to spinal cord motor neurons [25]. Alterations in LKB1 activity may therefore account for the STK11 SNP being a risk factor for MS.

Our previous study examined STK11 SNP prevalence in US patients PwMS of European descent. To address whether this SNP is a risk factor in other populations, we examined its frequency in PwMS recruited at multiple Veterans Affairs Medical Centers (VAMCs) and compared that to its frequency in samples from PwMS and controls collected at academic institutes and a non-for-profit MS tissue repository. Previous epidemiological studies have suggested increased risk and greater disease severity of MS in military veterans compared to civilians [26-31]; however, whether genetic factors contribute to those differences has not been addressed. Our findings show that the STK11 SNP is a risk factor for AA SPMS patients; and in contrast to White PWMS where the age at first symptom onset and diagnosis is younger in SNP carriers, in AA PWMS it is associated with older ages.

\section{Methods and Materials}

\section{Study Population}

The study population was comprised of 5 distinct cohorts (Table 1). Plasma samples $(n=91)$ were obtained from AA and White PwMS who participated in the VA Longitudinal MS Study (VALOMS) by the VA MS Centers of Excellence [32], and used to isolate genomic DNA (gDNA). Informed consent was obtained from subjects between the ages of 18 and 65 years at enrollment; and had a diagnosis of definite MS based on McDonald Criteria [33,34]. gDNA samples $(n=109)$ from AA PwMS were also obtained from the Accelerated Cure Project for Multiple Sclerosis (ACP) Repository (Waltham, MA). Full details of that study are available at www.acceleratedcure.org. gDNA samples from AA controls were provided by Dr. Oksenberg at the Department of Neurology, UCSF $(n=398)$ and from ACP $(n=42)$. 
Additional comparisons were made to previously described [17] cohorts of White PwMS $(n=654)$ and controls $(n=661)$ also from UCSF. Patients in all cohorts were treated with interferon-beta or glatiramer acetate which are known not to interfere with long-term progression or conversion to SPMS. All procedures were approved by the Baltimore VAMC Research and Development Committee, each participating VAMC, and the University of Maryland and of Illinois Institutional Review Boards.

\section{Genomic DNA extraction from plasma samples}

gDNA was extracted from plasma samples using Maxwell 16 LEV Blood DNA Kit (Promega, Madison, WI, \#AS1290). Concentrations were measured using Qubit 4 Fluorometer (ThermoFisher Scientific, Waltham, MA) or Quantus Fluorometer (Promega) calibrated with dsDNA (ThermoFisher Scientific). Samples with concentrations below $0.3 \mathrm{ng} / \mathrm{ul}$, or that were cloudy were amplified and repaired using the whole genome amplification REPLI-g FFPE Kit (Qiagen, Germantown, MD).

\section{DNA analysis}

Genotyping was performed using a TaqMan assay targeting the STK11 rs9282860 C/T allele (Life Technologies, Carlsbad, CA, \#C_25599132_10). PCRs were performed on an Applied Biosystems ViiA7 instrument (Waltham, MA) using TaqMan Genotyping Master Mix according to manufacturer's instructions. Data analysis was performed using ViiA7 software and Genotyper Software (Life Technologies). A subset of samples with either allele was validated by PCR amplification and Sanger sequencing.

\section{Data analysis}

Group means were compared using parametric independent sample T-tests for equal or non-equal variance based on results of Levene's test for variance. Odds ratios (ORs) were compared using Fisher's exact test and Bonferroni correction. All analyses were carried out using SPSS v28. MS Severity Scores (MSSS) were calculated from most recent Expanded Disability Status Scale scores (EDSS) and disease duration since symptom onset as described [35].

\section{Results}

\section{Demographics and Clinical Features of the VALOMS patients with MS}

gDNA samples were obtained from PwMS patients from 10 different VAMCs. The majority of AA PwMS $(n=33,36 \%)$ were from the Baltimore, MD and Washington, DC areas (Figure 1A) while the regional distribution of White PwMS ( $n=58,64 \%$ ) was more diverse (Figure 1B). The total cohort had 65 males and 26 females (2.5-fold ratio); in the AA cohort the gender ratio $(20 \mathrm{M} / 13 \mathrm{~F}$, ratio $=1.5)$ was lower than in the White cohort $(45 \mathrm{M} / 13 \mathrm{~F}$, ratio $=3.5)$. The age at initial exam (enrollment and blood draw) tended to be younger in AA than White PwMS (Table 1) and was significantly lower in AA compared to White male SPMS patients $(43.7 \pm 4.1$ vs $52.5 \pm 1.5)$. VALOMS SPMS patients tended to have an older age at initial exam than RRMS patients, which was significantly different between White SPMS and RRMS females patients $(52.6 \pm 1.8$ vs $42.8 \pm 2.8)$.

The age at diagnosis (Figure 2A, Table S1) was younger in all AA compared to all White PwMS $(36.2 \pm 1.7$ vs $41.2 \pm 1.3)$ but did not differ between the individual AA versus White genders. The AA male SPMS, but not RRMS patients had a younger age at diagnosis than corresponding White SPMS patients (35.6 \pm 4.4 vs $44.2 \pm 1.9$ ), as did the combined male and female AA versus White SPMS patients 
(36.8 \pm 3.3 vs $44.1 \pm 1.6)$. White SPMS patients tended to have an older age at diagnosis than White RRMS patients which was significantly different between the combined male and female groups $(44.1 \pm 1.6$ vs $38.7 \pm 2.0)$. In contrast to age of diagnosis, the age at first symptom onset was similar between all VALOMS AA and White PwMS (Figure 2B).

As expected, in both White and AA groups, the EDSS scores were significantly higher in SPMS patients than corresponding RRMS patients (Table S1, Figure 3A), except for the female white cohort. Interestingly, AA SPMS patients tended to have higher EDSS scores than White SPMS patients, being significantly different for combined male and female patients ( $6.3 \pm 0.3$ vs $5.3 \pm 0.3$ ). As for EDSS, MSSS, a measure of disease severity taking into account duration, was higher in SPMS compared to RRMS patients (Figure 3B), and AA SPMS patients tended to have higher MSSS than White SPMS patients with a significant difference for combined male and female cohorts $(7.4 \pm 0.4$ versus $5.8 \pm 0.5)$.

\section{Comparison of VALOMS patients with MS to other cohorts}

Comparisons were made to determine if MS diagnosis, treatment or progression differed between PwMS seen at VAMCs compared to non-VAMC facilities. We first compared AA PwMS from the VALOMS cohort to AA PWMS recruited by ACP from MS clinics across the US. The geographic distribution of the ACP cohort differed from that of the VALOMS group, with the majority of ACP subjects from Georgia (Figure 1C). In contrast to the VALOMS cohort, the ACP cohort consisted only of RRMS patients and was mostly females $(92 \mathrm{~F} / 17 \mathrm{M}$, ratio $=5.4)$. Although these were RRMS patients, comparisons were made to VALOMS SPMS patients since SPMS begins as a RR disease. There were no significant differences between the average age at exam (Table 1), age at diagnosis, disease duration from diagnosis, or EDSS scores (Table S1; Figure 3A) between the 2 groups; although as expected, VALOMS SPMS patients had higher EDSS scores than the ACP RRMS patients.

Comparisons of the VALOMS White PWMS were made to a previously described UCSF cohort of 654 RRMS White PwMS of European descent [17], recruited from 44 different US states [9]. The age at exam was similar between the groups (Table 1), although slightly higher in the VALOMS White SPMS males than UCSF White RRMS male patients ( $52.5 \pm 1.5$ vs $46.0 \pm 0.8)$. VALOMS White SPMS patients tended to have older age of symptom onset than the UCSF RRMS patients with significant differences between male patients $(39.2 \pm 2.3$ versus $32.9 \pm 0.6)$ and between the combined male and female groups $(36.9 \pm 2.1$ versus $32.7 \pm 0.4)$ (Table S1, Figure 2B). In addition, the VALOMS White SPMS female patients had significantly longer disease duration than the female UCSF White RRMS patients. The VALOMS White SPMS patients had higher EDSS scores (Figure 3A) and showed a trend toward higher MSSS (Figure 3B) however small groups sizes prevented statistical significance except for the combined male and female cohorts.

\section{Comparison of STK11 SNP prevalence in AA patients with MS to controls}

The prevalence of the STK11 SNP in AA subjects was not significantly different between controls from the ACP and UCSF cohorts so these were combined to yield 440 total controls. Comparison of the VALOMS AA PwMS to controls showed no significant difference in STK11 SNP prevalence (Table 2, Figure 4) although there was a trend toward greater prevalence in female patients (4/13, 31\% genotype frequency) than in female controls $(43 / 329,13 \%$; $O R=2.9, p=0.088)$. When MS subtype was taken into consideration, the difference between all SPMS patients (4/12, 33\%) and controls (55/440,13\%) almost reached statistical significance $(O R=3.5, p=0.058)$. To gain further power, we combined the VALOMS 
and ACP AA PwMS cohorts. Comparison to the AA controls yielded similar results as using the VALOMS group alone, with no significant difference in STK11 SNP prevalence between all AA PwMS patients and all controls; nor any difference in RRMS patients versus controls. Since the ACP cohort contained only RRMS patients, differences between SPMS patients and controls remained unchanged.

\section{Comparison of STK11 SNP prevalence in AA to White patients with MS}

Although the STK11 SNP prevalence in the VALOMS AA PwMS was greater than in the VALOMS White PwMS (Table 2, Figure 4), group sizes were too small to reach statistical significance, except for the prevalence in all AA SPMS patients $(4 / 12,33 \%)$ which was significantly greater than the prevalence in all White SPMS patients $(1 / 28,4 \%$; OR=13.5, $p=0.022)$. STK11 SNP prevalence tended to be greater in VALOMS compared to ACP AA PwMS, however only the difference between all VALOMS SPMS and all ACP RRMS patients was close to being significant (OR=3.7, $p=0.065)$. Comparison to the UCSF White PWMS [17] showed higher STK11 SNP prevalence in all AA (7/33, $21 \%)$ compared to all White PwMS (68/664,10.4\%), however none of those differences were statistically significant. The higher prevalence of the SNP in female AA PwMS (4/13,31\%) compared to female White PwMS (49/445, 11.0\%) was close to significant $(\mathrm{OR}=3.6, \mathrm{p}=0.052)$.

\section{Comparison of STK11 SNP prevalence in VALOMS patients with MS to controls}

Comparison of VALOMS, ACP, or combined VALOMS and ACP AA PWMS to AA controls did not show any statistical difference in STK11 SNP prevalence; however the prevalence in the VALOMS SPMS $(4 / 12,33 \%)$ group almost reached significance when compared to all AA controls $(55 / 440,13 \%$, $\mathrm{OR}=3.5, \mathrm{p}=0.058$ ). Similarly, although the SNP prevalence tended to be higher in VALOMS White RRMS patients compared to the White controls (Table 2, Figure 4), no significant differences were detected. This contrasts to UCSF White RRMS patients who had significantly increased STK11 SNP prevalence compared to White controls with an OR of 1.6 in females [17].

\section{The STK11 SNP is associated with age at first symptom onset and at diagnosis}

In the VALOMS AA PwMS cohort, neither the average EDSS, age at first symptom onset, disease duration, nor MSSS (Table 3, Figure 5) was significantly different between subjects with the STK11 SNP and those without; however, the age at diagnosis tended to be older and was almost significantly different in the combined male and female cohort $(41.9 \pm 4.1$ versus $34.6 \pm 1.7, p=0.073)$ in subjects who carried the SNP. In the combined cohort of VALOMS and ACP AA PwMS, the average age at diagnosis was older in those with the SNP compared to those without $(41.2 \pm 2.5$ versus $35.2 \pm 0.8$, $\mathrm{p}<0.05)$, and this association was also present in male, but not female subjects with the SNP (47.6 \pm 4.9 versus $34.7 \pm 1.5, p<0.01)$. When stratified by MS subtype, age at first symptom onset and at diagnosis were both significantly older in SPMS subject with the SNP, however there were no significant associations of these parameters with the SNP in RRMS subjects.

In contrast to AA PwMS, White PwMS in the VALOMS cohort with the STK11 SNP (Table 4) tended to have a younger, rather than older age at first symptom onset and at diagnosis, and lower EDSS and MSSS; however, none of those differences reached statistical significance. In contrast, in the UCSF White RRMS subject [17], those with the SNP had significantly lower EDSS, and male subject showed a trend to having a younger age at first symptom onset. 


\section{Discussion}

In the current study we examined the prevalence of the STK11 SNP in AA and White PwMS enrolled in the VALOMS study, as well as corresponding cohorts recruited from non-VAMC facilities. Comparison of clinical features (age at first symptom onset, age at diagnosis, EDSS, and MSSS) showed relatively little differences between the VALOMS patients and the other cohorts, although as expected, SPMS patients had higher neurological scores. The STK11 SNP was also present at higher prevalence in AA SPMS patients, and was close to be significantly greater than that in AA controls, suggesting that it may be a risk factor for SPMS in this population. Interestingly, AA SPMS patients with the SNP had an older age of first symptom onset and diagnosis that those with the common allele; suggesting an influence of this SNP at early (onset) as well as late (conversion) stages of MS disease.

\section{MS genetic risk factors and identification of STK11 SNP}

It is now accepted that multiple factors contribute to the causes of MS, including genetic and environmental influences [36,37]. While genomic variants in the MHC locus represent the highest risk factors for MS [3], GWAS studies have identified over 200 non HLA variants [1], which together with MHC variants may account for $25-50 \%$ of the total genetic risk. However, large GWAS studies have typically been restricted to common variants, e.g. those present in the general Caucasian population at a minor allele frequency (MAF) of $5 \%$ or greater, or to rare variants $(0.01-5 \%$ MAF) present in coding regions [2]. This approach therefore can overlook SNPs present in non-coding regions at lower prevalence but with higher risk. The STK11 SNP, originally identified in a family in which 5 siblings had been diagnosed with MS or CIS [17] may have been overlooked since in healthy European populations, the MAF ranges from slightly over $1 \%$ (Finish, Great Britain) to slightly over $9 \%$ in Italian populations for an average MAF of $3.9 \%$ for Europeans [38]. It is also possible that the stringent criteria of large GWAS are designed to find risk factors, but not factors associated with clinical progression.

The functional consequences of the STK11 SNP are yet to be fully determined; however it is likely that any alterations in LKB1 expression or activity in multiple cell types could contribute to increased MS risk or progression. LKB1 depletion from spinal cord neurons led to axonal degeneration, macrophage infiltration, and hindlimb paralysis [39]; mice with LKB1 depletion from regulatory Tcells developed an early onset autoimmune disease [40]; and depletion from B-lymphocytes led to their spontaneous activation [41]. We showed that conditional knockout of LKB1 from astrocytes worsened EAE disease severity, associated with increased glial inflammation, increased MHC Class-II expression, greater numbers of Th17 type T-lymphocytes, reduced mitochondrial function, and increased spinal cord motor neuron damage [25]. Whether similar events occur in PwMS harboring this SNP is not yet known, although preliminary data (AIB, unpublished) suggest LKB1 mRNA levels are decreased in PBMCs from MS patients with the SNP.

\section{Military Veterans and MS}

It was reported that the incidence and severity of MS is higher in Veteran populations compared to civilians with the highest incidence in AAs [28]. A study of 2,691 Veterans with MS who had active duty during the Gulf War Era [28] revealed no significant effect of race or sex on the average age of symptom onset (30.7 \pm 7.6 for the entire group). Consistent with that, we found no effect of race or sex on age of first symptom onset in the VALOMS cohort. However, we found the age at diagnosis tended to be younger in AA than White PwMS, with a significant difference between SPMS cohorts. We also found 
MSSS tended to be higher in AA than White PwMS, however only the difference between SPMS patients reached statistical significance. This contrasts from a previous study which found that AA male Veterans, a cohort comprised mainly of RRMS cases, had higher MSSS than other sex-race groups [30]. However, that finding may be due to inclusion of PPMS patients in the AA subgroup, which comprised $9.1 \%$ of all AA patients, compared to $5.1 \%$ PPMS in the White cohort; in contrast PPMS cases were not included in the current study. As expected, EDSS scores and MSSS were higher in SPMS than RRMS patients in both AA and White patients. Overall, our results do not indicate any significant differences in disease onset or severity in AA compared to White Veterans, other than that expected for SPMS cohorts.

Comparison of the AA VALOMS to ACP AA RRMS patients showed no significant differences in either age of exam, age at diagnosis, or EDSS scores; although disease duration tended to be longer in VALOMS subjects. Similarly, there were no significant differences between VALOMS White RRMS and the UCSF White RRMS cohorts. This suggests that Veteran PWMS at VAMCs are seen, diagnosed, and have similar disease progression as patients treated at non-VA facilities.

\section{STK11 SNP prevalence in VALOMS PwMS}

We found that the STK11 SNP prevalence tended to be higher in VALOMS AA PwMS than AA controls, with an almost significant difference $(P=0.058)$ for SPMS patients (Table 2, Figure 4). The minor allele frequency (MAF) of the SNP in AA controls was $6.7 \%$, similar to a MAF of $6.8 \%$ reported in the 1000 genome database for all African populations (AFR, $n=661$ ) [38], although the MAF for the smaller American African Southwest sub-cohort (ASW, $n=61$ ) is lower $(4.9 \%)$. A more representative study of the AA population in the US (ALFA, $n=1977$ ) reported a MAF of $6.1 \%$ [42] similar to what we observed in AA controls. STK11 MAFs were higher in the VALOMS AA PWMS, ranging from $4.0 \%$ in RRMS males, to $25.0 \%$ in SPMS females, and averaging $11.9 \%$ for all patients. The STK11 MAF in the ACP PwMS was lower (6.3\%); this may reflect regional differences in the genetic makeup of the ACP cohort (mostly from Southern US) compared to the VALOMS cohort (mostly from Eastern US regions). Interestingly, the SNP prevalence in AA SPMS patients was significantly greater compared to White SPMS patients $(\mathrm{OR}=13.5, \mathrm{P}=0.022)$. In contrast, the STK11 SNP prevalence in VALOMS White PwMS was not significantly different from that in UCSF White controls.

\section{STK11 and MS severity and progression}

Although both EDSS scores and MSSS tended to be higher in AA subjects who harbored the STK11 SNP compared to those with the C/C allele (Table 3), none of those differences reached statistical significance. This contrasts from lower MSSS and EDSS scores in the VALOMS White PWMS, and from a significant association of the SNP with lower EDSS scores in UCSF White PwMS [17]. In the VALOMS AA PwMS, the SNP was associated with older age at disease onset and at diagnosis in all cohorts and these were significantly different in SPMS patients. In contrast, there was a trend towards an association of the SNP with younger age at onset in the UCSF White RRMS patients, which we also observed in the VALOMS White cohorts (Table 4). While small group sizes may account for lack of significant effects in the VALOMS cohorts, the trend towards an opposite association with MSSS, EDSS, disease onset, and disease diagnosis suggests that the variant differentially influences disease progression in AA compared to White PwMS. 
Despite the small group size in the AA SPMS cohort ( $n=12$ total), the STK11 SNP was almost significantly associated with MS risk compared to controls; and significantly associated when compared to White SPMS patients. This SNP may therefore represent a risk factor for developing SPMS in the AA population. In this regard, relatively few studies have attempted to identify risk factors for either MS progression or conversion from one form to another (e.g. CIS to MS; or RRMS to SPMS) [43]. Environmental and dietary factors may contribute to conversion, including deficiencies in vitamin $D$ which may be a risk for conversion of CIS to MS [44] and rare variants in cytochrome CYP27B1 which converts Vitamin D to its active form have also been identified as MS risk factors $[45,46]$. Smoking has a significant association with overall MS risk as well as SPMS risk, but not CIS to MS conversion [47].

Several genetic studies have attempted to identify SNPs associated with disease progression or conversion. In contrast to the strong association of HLA with MS risk, several studies were unable to find a strong influence of HLA on MS type, progression, relapse rate, or severity [48], although that women with high HLA burden developed MS at a younger age [49]. More recently, a retrospective study of 1295 White PwMS revealed an association of the HLA-A*02:01 allele with decreased conversion to SPMS [50]. In a GWAS looking at conversion from the first demyelinating event to MS, there were 2 non-HLA and 1 HLA SNP identified which predicted both conversion and relapse; as well as 3 SNPs that predicted only conversion and 3 that predicted only relapse [51]. A variant in the gene encoding AHI1 (Abelson Helper Integration Site 1, involved in cell maturation and proliferation) is associated with increased relapse rate in patients homozygous for the variant [52]; and a SNP in the neuropeptide galanin gene was associated with more rapid disease progression (MSSS) [53]. In a comparison of 'benign' MS (EDSS <= 3 after 15 or more years) to 'aggressive' MS (EDSS >= 6 within 5 years of onset) 2 SNPs were found associated with disease course, one in CPXM2 (carboxypeptidase X) associated with an aggressive course; and one in IGSF9B (immunoglobulin superfamily member) with a benign course, as well as trend for a $3^{\text {rd }}$ SNP with benign disease in inflammasome protein NLRP9 (nucleotidebinding domain-like receptor protein 9) [54]. Variants in NLRP3 as well as interleukin-1b have also been associated with increased severity and faster progression [55].

The development of SPMS has been associated with mitochondrial dysfunction and production of reactive oxygen species [56,57]. In one large study, certain mitochondrial haplotypes were found associated with increased MS risk [58], with many SNPs present in genes encoding mitochondrial complex I. A search for variants that could distinguish different MS forms [59] compared variants across PPMS, RRMS, and SPMS patients. Of 4 variants identified in PPMS, 3 were related to HSP (hereditary spastic paraplegia), a disease of upper motor neurons involving metabolic disturbance [60], further screening showed enrichment for HSP-related variants in SPMS but not RRMS patients. Moreover, 2 variants, REEP1 and SPG7, are involved in mitochondrial function. REEP1 plays a role in endoplasmic reticulum interactions with mitochondria and can cause calcium overload and axonal injury [61]; while the inner mitochondrial protein SPG7 variants can cause deficiency in complex I [62]. Further support that mitochondrial dysfunction contributes to disease progression comes from EAE studies [63] which show that the xanthine oxidase inhibitor febuxostat protects neurons by increasing mitochondrial protein expression and ATP generation. In our EAE studies [25] we showed that conditional depletion of LKB1 from astrocytes, which can provide energy substrates to neurons, reduced astrocyte mitochondrial complex expression, and reduced expression of mRNAs related to astrocyte metabolism. In vitro, LKB1 depletion from astrocytes reduced expression of metabolic-related mRNAs and increased 
astrocyte inflammatory responses. This suggests that in PwMS, the STK11 SNP could decrease mitochondrial function or glial metabolism, either which could contribute to increased risk of SPMS.

We found that the STK11 SNP was associated with an older age at symptom onset in the AA SPMS patients (Table 3). The association of older age of onset and being male with SPMS was reported in 2 large studies [50,64], as well as increased risk of SPMS with older age at symptom onset, higher EDSS and faster disability accrual [64]. In contrast, although differences did not reach statistical significance, the STK11 SNP was associated with a younger age of onset in AA RRMS patients; as well as in the VALOMS and UCSF White RRMS patients. Since SPMS begins as a RR disease, the age at first symptom onset in SPMS patients reflects the age at which RRMS began. A possible explanation to reconcile being a risk factor for MS, yet associated with delayed onset in AA SPMS patients is that the SNP, which is ubiquitously expressed throughout the body, may act to suppress the early development of MS, for example by increasing apoptosis of Tcell populations that contribute to disease onset. after disease onset, its actions in other cell types, for example by reducing mitochondrial function in neurons, accelerates progression from RRMS to SPMS.

The current study has several limitations. Relatively small group sizes limit the ability to detect statistically significant differences in demographic or clinical features; although an almost significant increase in STK11 SNP prevalence in AA SPMS patients versus controls was achieved with 12 SPMS patients; replication in a larger cohort is required. The geographic distribution of subjects also differs; whereas VALOMS AA PWMS were primarily from Baltimore, MD and Washington, DC areas, AA PwMS in the ACP cohort were predominantly from Southern US, while AA controls were drawn from across 39 different US States [9]. Since the AA genetic makeup differs across the US [12,65], comparison of cohorts from different regions may be confounded by differences in African versus European ancestry.

To our knowledge, the current study represents one of the first examinations of non-HLA SNPs in a demographically diverse cohort that included military Veterans, a population with racial and ethnic diversity that reflects the US population. These findings, therefore, may be more relevant to the US population than results from larger GWAS discovery studies which primarily use Caucasian cohorts of European descent. Further study of the STK11 SNP is therefore warranted in large population-based cohorts to assess for associations with morbidity and mortality.

Data availability. All data will be made available upon request after publication. 


\section{Abbreviations}

$\begin{array}{ll}\text { AA } & \text { African American } \\ \text { CIS } & \text { Clinically isolated syndrome } \\ \text { EAE } & \text { Experimental autoimmune encephalomyelitis } \\ \text { EDSS } & \text { Expanded disability severity score } \\ \text { GWAS } & \text { Genome wide array study } \\ \text { LKB1 } & \text { Liver kinase B1 } \\ \text { MAF } & \text { Minor allele frequency } \\ \text { MHC } & \text { Major histocompatibility } \\ \text { MSSR } & \text { MS Surveillance Registry } \\ \text { MSSS } & \text { MS severity score } \\ \text { mtDNA } & \text { Mitochondrial DNA } \\ \text { OR } & \text { Odds Ratio } \\ \text { PPMS } & \text { Primary progressive MS } \\ \text { PWMS } & \text { Patients with MS } \\ \text { RRMS } & \text { Relapsing-remitting MS } \\ \text { SNP } & \text { Single nucleotide polymorphism } \\ \text { SPMS } & \text { Secondary progressive MS } \\ \text { STK11 } & \text { Serine threonine kinase 11 } \\ \text { VALOMS } & \text { Veterans Affairs Longitudinal MS Study } \\ \text { VAMC } & \text { Veterans Affairs Medical Center }\end{array}$


Figure legends

\section{Figure 1. Regional distribution of VALOMS and ACP patients with MS}

Values for the number of male and female patients are shown by state for VALOMS (A) AA and (B) White PwMS; and (C) AA PwMS from ACP. nd, no data.

\section{Figure 2. Age at diagnosis of VALOMS AA and White patients with MS}

Average age at (A) diagnosis and (B) symptom onset for VALOMS AA (white bars), VALOMS White (black bars), ACP AA (gray bars in A), and UCSF White (gray bars in B) PwMS. Black lines indicate significance between groups $(P<0.05)$. Data are mean \pm se and are taken from Table 1 .

\section{Figure 3. MS disease severity in AA and White patients with MS}

Average (A) EDSS scores measured at last exam and (B) MSSS for VALOMS AA (white bars), VALOMS White (black bars), ACP AA (light gray bars in A), and UCSF White (dark gray bars in $\mathbf{A}$ and B) PwMS. Significance $(P<0.05)$ is indicated by black lines comparing VALOMS AA and White patients; gray lines comparing VALOMS to ACP AA PwMS; and dashed lines comparing VALOMS to UCSF White PwMS. Data is mean \pm se and are taken from Table 1 .

\section{Figure 4. STK11 SNP prevalence}

The minor allele frequency (MAF) for the $\mathrm{C} / \mathrm{T}$ genotype is shown for all groups. Significance $\left({ }^{*}, \mathrm{P}<0.05\right)$ is indicated by black lines; near significance is indicated by gray lines. Data are taken from Table 2.

\section{Figure 5. Effect of STK11 SNP on age at diagnosis and age at symptom onset}

Average (A) age at diagnosis and (B) age at symptom onset for VALOMS AA PWMS and for the combined VALOMS \& ACP AA PwMS (VA \& ACP), having the C/C (white bars) or C/T (black bars) allele. ${ }^{*}, \mathrm{P}<0.05 ;{ }^{* *}, \mathrm{P}<0.005$ versus $\mathrm{C} / \mathrm{C}$. Data is mean \pm se and are taken from Table 3. 
International_Multiple_Sclerosis_Genetics_Consortium. Multiple sclerosis genomic map implicates peripheral immune cells and microglia in susceptibility. Science 365, doi:10.1126/science.aav7188 (2019).

2 International_Multiple_Sclerosis_Genetics_Consortium. Low-Frequency and Rare-Coding Variation Contributes to Multiple Sclerosis Risk. Cell 175, 1679-1687.e1677, doi:10.1016/j.cell.2018.09.049 (2018).

3 Baranzini, S. E. \& Oksenberg, J. R. The Genetics of Multiple Sclerosis: From 0 to 200 in 50 Years. Trends Genet 33, 960-970, doi:10.1016/j.tig.2017.09.004 (2017).

4 Patsopoulos, N. A. et al. Genome-wide meta-analysis identifies novel multiple sclerosis susceptibility loci. Ann. Neurol 70, 897-912 (2011).

5 Beecham, A. H. et al. Analysis of immune-related loci identifies 48 new susceptibility variants for multiple sclerosis. Nat Genet 45, 1353-1360, doi:10.1038/ng.2770 (2013).

6 Isobe, N. et al. Genetic risk variants in African Americans with multiple sclerosis. Neurology 81, 219-227 (2013).

$7 \quad$ McElroy, J. P. et al. Refining the association of MHC with multiple sclerosis in African Americans. Hum Mol Genet 19, 3080-3088, doi:10.1093/hmg/ddq197 (2010).

8 Johnson, B. A. et al. Multiple sclerosis susceptibility alleles in African Americans. Genes Immun 11, 343-350, doi:10.1038/gene.2009.81 (2010).

9 Cree, B. A. et al. Modification of Multiple Sclerosis Phenotypes by African Ancestry at HLA. Arch Neurol 66, 226-233, doi:10.1001/archneurol.2008.541 (2009).

10 Oksenberg, J. R. et al. Mapping multiple sclerosis susceptibility to the HLA-DR locus in African Americans. Am J Hum Genet 74, 160-167, doi:10.1086/380997 (2004).

11 Beecham, A. H. et al. The genetic diversity of multiple sclerosis risk among Hispanic and African American populations living in the United States. Mult Scler 26, 1329-1339, doi:10.1177/1352458519863764 (2020).

12 Isobe, N. et al. Genetic burden in multiple sclerosis families. Genes Immun 14, 434-440 (2013).

13 Amezcua, L. \& McCauley, J. L. Race and ethnicity on MS presentation and disease course. Mult Scler 26, 561-567, doi:10.1177/1352458519887328 (2020).

14 Amezcua, L., Rivas, E., Joseph, S., Zhang, J. \& Liu, L. Multiple Sclerosis Mortality by Race/Ethnicity, Age, Sex, and Time Period in the United States, 1999-2015. Neuroepidemiology 50, 35-40, doi:10.1159/000484213 (2018).

15 Ventura, R. E., Antezana, A. O., Bacon, T. \& Kister, I. Hispanic Americans and African Americans with multiple sclerosis have more severe disease course than Caucasian Americans. Mult Scler 23, 1554-1557, doi:10.1177/1352458516679894 (2017).

16 Cree, B. A. et al. Clinical characteristics of African Americans vs Caucasian Americans with multiple sclerosis. Neurology 63, 2039-2045, doi:10.1212/01.wnl.0000145762.60562.5d (2004).

17 Boullerne, A. I. et al. A single-nucleotide polymorphism in serine-threonine kinase 11, the gene encoding liver kinase $\mathrm{B} 1$, is a risk factor for multiple sclerosis. ASN Neuro 7, doi:10.1177/1759091415568914 (2015).

18 Gan, R. Y. \& Li, H. B. Recent progress on liver kinase B1 (LKB1): expression, regulation, downstream signaling and cancer suppressive function. Int J Mol Sci 15, 16698-16718, doi:10.3390/ijms150916698 (2014).

19 Sebbagh, M., Olschwang, S., Santoni, M. J. \& Borg, J. P. The LKB1 complex-AMPK pathway: the tree that hides the forest. Fam. Cancer 10, 415-424 (2011).

20 Maclver, N. J. et al. The liver kinase B1 is a central regulator of T cell development, activation, and metabolism. J. Immunol 187, 4187-4198 (2011).

21 Chen, S. et al. Control of Treg cell homeostasis and immune equilibrium by Lkb1 in dendritic cells. Nat Commun 9, 5298, doi:10.1038/s41467-018-07545-8 (2018).

22 Beirowski, B. The LKB1-AMPK and mTORC1 Metabolic Signaling Networks in Schwann Cells Control Axon Integrity and Myelination: Assembling and upholding nerves by metabolic signaling in Schwann cells. Bioessays 41, e1800075, doi:10.1002/bies.201800075 (2019). 
23 Pooya, S. et al. The tumour suppressor LKB1 regulates myelination through mitochondrial metabolism. Nat. Commun 5, 4993 (2014).

24 Shen, Y. A. et al. Phosphorylation of LKB1/Par-4 establishes Schwann cell polarity to initiate and control myelin extent. Nat. Commun 5, 4991 (2014).

25 Kalinin, S. et al. Liver kinase B1 depletion from astrocytes worsens disease in a mouse model of multiple sclerosis. Glia 68, 600-616, doi:10.1002/glia.23742 (2020).

26 Wallin, M. T., Page, W. F. \& Kurtzke, J. F. Epidemiology of multiple sclerosis in US veterans. VIII. Long-term survival after onset of multiple sclerosis. Brain 123 ( Pt 8), 1677-1687 (2000).

27 Wallin, M. T., Page, W. F. \& Kurtzke, J. F. Multiple sclerosis in US veterans of the Vietnam era and later military service: race, sex, and geography. Ann Neurol 55, 65-71, doi:10.1002/ana.10788 (2004).

28 Wallin, M. T. et al. The Gulf War era multiple sclerosis cohort: age and incidence rates by race, sex and service. Brain 135, 1778-1785, doi:10.1093/brain/aws099 (2012).

29 Wallin, M. T. et al. Multiple sclerosis in gulf war era veterans. 2. Military deployment and risk of multiple sclerosis in the first gulf war. Neuroepidemiology 42, 226-234, doi:10.1159/000360701 (2014).

30 Wallin, M. T., Culpepper, W. J., Maloni, H. \& Kurtzke, J. F. The Gulf War era multiple sclerosis cohort: 3. Early clinical features. Acta Neurol Scand 137, 76-84, doi:10.1111/ane.12810 (2018).

31 Wallin, M. T. et al. The prevalence of MS in the United States: A population-based estimate using health claims data. Neurology 92, e1029-e1040, doi:10.1212/wnl.0000000000007035 (2019).

32 Royal, W. et al. Clinical and Demographic Features of Participants in a Veterans Affairs Longitudinal Study of Multiple Sclerosis. . Consortium of MS Centers Annual Meeting 14, 75-76 (2012).

33 Polman, C. H. et al. Diagnostic criteria for multiple sclerosis: 2005 revisions to the "McDonald Criteria". Ann Neurol 58, 840-846, doi:10.1002/ana.20703 (2005).

34 Polman, C. H. et al. Diagnostic criteria for multiple sclerosis: 2010 revisions to the McDonald criteria. Ann Neurol 69, 292-302, doi:10.1002/ana.22366 (2011).

35 Roxburgh, R. H. et al. Multiple Sclerosis Severity Score: using disability and disease duration to rate disease severity. Neurology 64, 1144-1151, doi:10.1212/01.wnl.0000156155.19270.f8 (2005).

36 Kildebeck, E. J. et al. The emergence of neuroepidemiology, neurovirology and neuroimmunology: the legacies of John F. Kurtzke and Richard 'Dick' T. Johnson. J Neurol 264, 817-828, doi:10.1007/s00415-016-8293-y (2017).

37 Olsson, T., Barcellos, L. F. \& Alfredsson, L. Interactions between genetic, lifestyle and environmental risk factors for multiple sclerosis. Nat Rev Neurol 13, 25-36, doi:10.1038/nrneurol.2016.187 (2017).

38 Howe, K. L. et al. Ensembl 2021. Nucleic Acids Res, doi:10.1093/nar/gkaa942 (2020).

39 Sun, G., Reynolds, R., Leclerc, I. \& Rutter, G. A. RIP2-mediated LKB1 deletion causes axon degeneration in the spinal cord and hind-limb paralysis. Dis. Model. Mech 4, 193-202 (2011).

$40 \mathrm{Wu}, \mathrm{D}$. et al. Lkb1 maintains Treg cell lineage identity. Nat Commun 8, 15876, doi:10.1038/ncomms15876 (2017).

41 Walsh, N. C. et al. LKB1 inhibition of NF-kappaB in B cells prevents T follicular helper cell differentiation and germinal center formation. EMBO Rep 16, 753-768, doi:10.15252/embr.201439505 (2015).

42 Phan, L. et al. ALFA: Allele Frequency Aggregator. National Center for Biotechnology Information www.ncbi.nlm.nih.gov/snp/docs/gsr/alfal (2020).

43 Jokubaitis, V. G. \& Butzkueven, H. A genetic basis for multiple sclerosis severity: Red herring or real? Mol Cell Probes 30, 357-365, doi:10.1016/j.mcp.2016.08.007 (2016).

44 Ascherio, A. et al. Vitamin D as an Early Predictor of Multiple Sclerosis Activity and Progression. JAMA Neurol (2014).

45 Ascherio, A. Environmental factors in multiple sclerosis. Expert. Rev. Neurother 13, 3-9 (2013). 
46 Munger, K. L. \& Ascherio, A. Prevention and treatment of MS: studying the effects of vitamin D. Mult Scler 17, 1405-1411, doi:10.1177/1352458511425366 (2011).

47 Degelman, M. L. \& Herman, K. M. Smoking and multiple sclerosis: A systematic review and meta-analysis using the Bradford Hill criteria for causation. Mult Scler Relat Disord 17, 207-216, doi:10.1016/j.msard.2017.07.020 (2017).

48 Longbrake, E. E. \& Hafler, D. A. Linking Genotype to Clinical Phenotype in Multiple Sclerosis: In Search of the Holy Grail. JAMA Neurol 73, 777-778, doi:10.1001/jamaneurol.2016.1227 (2016).

49 Isobe, N. et al. Association of HLA Genetic Risk Burden With Disease Phenotypes in Multiple Sclerosis. JAMA Neurol 73, 795-802, doi:10.1001/jamaneurol.2016.0980 (2016).

50 Misicka, E., Sept, C. \& Briggs, F. B. S. Predicting onset of secondary-progressive multiple sclerosis using genetic and non-genetic factors. J Neurol 267, 2328-2339, doi:10.1007/s00415020-09850-z (2020).

51 Pan, G. et al. Role of genetic susceptibility variants in predicting clinical course in multiple sclerosis: a cohort study. J Neurol Neurosurg Psychiatry 87, 1204-1211, doi:10.1136/jnnp-2016313722 (2016).

52 Graves, J. S. et al. The multiple sclerosis risk allele within the AHI1 gene is associated with relapses in children and adults. Mult Scler Relat Disord 19, 161-165, doi:10.1016/j.msard.2017.10.008 (2018).

53 Lioudyno, V. et al. The effect of galanin gene polymorphism rs948854 on the severity of multiple sclerosis: A significant association with the age of onset. Mult Scler Relat Disord 37, 101439, doi:10.1016/j.msard.2019.101439 (2020).

54 Gil-Varea, E. et al. Exome sequencing study in patients with multiple sclerosis reveals variants associated with disease course. J Neuroinflammation 15, 265, doi:10.1186/s12974-018-1307-1 (2018).

55 Soares, J. L., Oliveira, E. M. \& Pontillo, A. Variants in NLRP3 and NLRC4 inflammasome associate with susceptibility and severity of multiple sclerosis. Mult Scler Relat Disord 29, 26-34, doi:10.1016/j.msard.2019.01.023 (2019).

56 Campbell, G. R., Worrall, J. T. \& Mahad, D. J. The central role of mitochondria in axonal degeneration in multiple sclerosis. Mult Scler 20, 1806-1813, doi:10.1177/1352458514544537 (2014).

57 Campbell, G. R. et al. Mitochondrial DNA deletions and neurodegeneration in multiple sclerosis. Ann Neurol 69, 481-492, doi:10.1002/ana.22109 (2011).

58 Tranah, G. J. et al. Mitochondrial DNA sequence variation in multiple sclerosis. Neurology 85, 325-330, doi:10.1212/wnl.0000000000001744 (2015).

$59 \mathrm{Jia}, \mathrm{X}$. et al. Genome sequencing uncovers phenocopies in primary progressive multiple sclerosis. Ann Neurol 84, 51-63, doi:10.1002/ana.25263 (2018).

60 Blackstone, C. Hereditary spastic paraplegia. Handb Clin Neurol 148, 633-652, doi:10.1016/b978-0-444-64076-5.00041-7 (2018).

61 Zheng, P. et al. DNA damage triggers tubular endoplasmic reticulum extension to promote apoptosis by facilitating ER-mitochondria signaling. Cell Res 28, 833-854, doi:10.1038/s41422018-0065-z (2018).

62 Atorino, L. et al. Loss of m-AAA protease in mitochondria causes complex I deficiency and increased sensitivity to oxidative stress in hereditary spastic paraplegia. J Cell Biol 163, 777787, doi:10.1083/jcb.200304112 (2003).

63 Honorat, J. A. et al. Febuxostat ameliorates secondary progressive experimental autoimmune encephalomyelitis by restoring mitochondrial energy production in a GOT2-dependent manner. PLoS One 12, e0187215, doi:10.1371/journal.pone.0187215 (2017).

64 Fambiatos, A. et al. Risk of secondary progressive multiple sclerosis: A longitudinal study. Mult Scler 26, 79-90, doi:10.1177/1352458519868990 (2020).

65 Adhikari, K., Chacón-Duque, J. C., Mendoza-Revilla, J., Fuentes-Guajardo, M. \& Ruiz-Linares, A. The Genetic Diversity of the Americas. Annu Rev Genomics Hum Genet 18, 277-296, doi:10.1146/annurev-genom-083115-022331 (2017). 

Table 1: Study Cohorts and Age at Exam

\begin{tabular}{|r|cll|c|l|}
\hline Source & Race & Gender & MS Type & N & Age at Exam \\
\hline VALOMS & AA & Male & RR & 13 & $44.6 \pm 2.4$ \\
& & & SP & 7 & $43.7 \pm 4.1 \mathbf{~ a ~}$ \\
\cline { 3 - 6 } & & Female & RR & 8 & $41.1 \pm 3.0$ \\
& & & SP & 5 & $48.0 \pm 4.5$ \\
\cline { 2 - 6 } & White & Male & RR & 24 & $48.7 \pm 2.1$ \\
& & & SP & 21 & $52.5 \pm 1.5 \mathbf{b}$ \\
\cline { 2 - 6 } & & Female & RR & 6 & $42.8 \pm 2.8 \mathbf{c}$ \\
& & & SP & 7 & $52.6 \pm 1.6$ \\
\hline ACP & AA & Male & RR & 17 & $40.4 \pm 2.7$ \\
& & Female & RR & 92 & $42.3 \pm 1.1$ \\
\hline UCSF & White & Male & RR & 209 & $46.0 \pm 0.8$ \\
& & Female & RR & 445 & $47.0 \pm 2.2$ \\
\hline ACP & AA & Male & Control & 11 & $49.9 \pm 4.1$ \\
& & Female & Control & 31 & $46.9 \pm 3.1$ \\
\hline UCSF & AA & Male & Control & 100 & $45.3 \pm 1.1$ \\
& & Female & Control & 298 & $43.5 \pm 0.6$ \\
\hline ACP \& UCSF & AA & Male & Control & 111 & $45.8 \pm 1.1$ \\
& & Female & Control & 329 & $43.8 \pm 0.6$ \\
\hline UCSF & White & Male & Control & 236 & $44.5 \pm 0.9$ \\
& & Female & Control & 425 & $42.6 \pm 0.6$ \\
\hline
\end{tabular}

$a, P<0.05$ versus VA White male SPMS; $b, P<0.05$ versus UCSF White male RRMS; $c, P<0.05$ versus VA White female SPMS. Data is mean \pm se. AA, African American; ACP, Accelerated Cure Project; RR, relapsing remitting; SP, secondary progressive. 
Table 2: Prevalence of STK11 SNP in case and control groups

\begin{tabular}{|c|c|c|c|c|c|c|c|c|c|c|c|c|}
\hline \multirow[b]{2}{*}{ Cohort } & \multirow[b]{2}{*}{ Race } & \multirow[b]{2}{*}{ Gender } & \multirow[b]{2}{*}{$\begin{array}{l}\text { MS } \\
\text { Type }\end{array}$} & \multicolumn{2}{|c|}{ STK11 Allele } & \multirow[b]{2}{*}{ Genotype } & \multirow[b]{2}{*}{ MAF } & \multicolumn{5}{|c|}{ Odds Ratio vs: } \\
\hline & & & & $\mathrm{C} / \mathrm{T}$ & $\mathrm{C} / \mathrm{C}$ & & & $\begin{array}{l}\text { VA White } \\
\text { MS }\end{array}$ & $\begin{array}{l}\text { ACP } \\
\text { MS }\end{array}$ & $\begin{array}{l}\text { AA } \\
\text { Ctl }\end{array}$ & $\begin{array}{c}\text { UCSF White } \\
\text { MS }\end{array}$ & $\begin{array}{c}\text { USCF White } \\
\text { Ctl }\end{array}$ \\
\hline \multirow{18}{*}{ VALOMS } & \multirow[t]{9}{*}{ AA } & \multirow[t]{3}{*}{ Male } & RR & 1 & 12 & $8 \%$ & $4.0 \%$ & 0.6 & 0.6 & 0.7 & 0.8 & \\
\hline & & & SP & 2 & 5 & $29 \%$ & $16.7 \%$ & 8.0 & 3.0 & 3.3 & 4.0 & \\
\hline & & & Both & 3 & 17 & $15 \%$ & $8.1 \%$ & 1.8 & 1.3 & 1.5 & 1.8 & \\
\hline & & \multirow[t]{3}{*}{ Female } & RR & 2 & 6 & $25 \%$ & $14.3 \%$ & 1.7 & 2.5 & 2.2 & 2.7 & \\
\hline & & & SP & 2 & 3 & $40 \%$ & $25.0 \%$ & $\mathrm{n} / \mathrm{a}$ & 4.9 & 4.4 & 5.4 & \\
\hline & & & Both & 4 & 9 & $31 \%$ & $18.2 \%$ & 5.3 & 3.3 & 2.9 & $3.6^{c}$ & \\
\hline & & \multirow[t]{3}{*}{ Both } & RR & 3 & 18 & $14 \%$ & $7.7 \%$ & 1.1 & 1.2 & 1.2 & 1.4 & \\
\hline & & & SP & 4 & 8 & $33 \%$ & $20.0 \%$ & $13.5^{\mathrm{a}}$ & $3.7^{\mathrm{e}}$ & $3.5^{b}$ & 4.3 & \\
\hline & & & Both & 7 & 26 & $21 \%$ & $11.9 \%$ & 2.9 & 2.0 & 1.9 & 2.3 & \\
\hline & \multirow[t]{9}{*}{ White } & \multirow[t]{3}{*}{ Male } & $\mathbf{R R}$ & 3 & 21 & $13 \%$ & $6.7 \%$ & & & & 1.4 & 1.4 \\
\hline & & & SP & 1 & 20 & $5 \%$ & $2.4 \%$ & & & & 0.5 & 0.5 \\
\hline & & & Both & 4 & 41 & $9 \%$ & $4.7 \%$ & & & & 1.0 & 1.0 \\
\hline & & \multirow[t]{3}{*}{ Female } & $\mathbf{R R}$ & 1 & 5 & $17 \%$ & $9.1 \%$ & & & & 1.6 & 2.1 \\
\hline & & & SP & 0 & 7 & $0 \%$ & $0.0 \%$ & & & & 1.0 & 0.0 \\
\hline & & & Both & 1 & 12 & $8 \%$ & $4.0 \%$ & & & & 1.5 & 1.0 \\
\hline & & \multirow[t]{3}{*}{ Both } & RR & 4 & 26 & $13 \%$ & $7.1 \%$ & & & & 1.3 & 1.7 \\
\hline & & & SP & 1 & 27 & $4 \%$ & $1.8 \%$ & a & & & 0.3 & 0.4 \\
\hline & & & Both & 5 & 53 & $9 \%$ & $4.5 \%$ & & & & 1.2 & 1.1 \\
\hline \multirow{3}{*}{ ACP } & \multirow[t]{3}{*}{ AA } & Male & $\mathbf{R R}$ & 2 & 15 & $12 \%$ & $6.3 \%$ & & & 1.1 & & \\
\hline & & Female & RR & 11 & 81 & $12 \%$ & $6.4 \%$ & & & 0.9 & & \\
\hline & & Both & $\mathbf{R} \mathbf{R}$ & 13 & 96 & $12 \%$ & $6.3 \%$ & & $\mathrm{e}$ & 0.9 & & \\
\hline \multirow{3}{*}{$\begin{array}{c}\text { VALOMS \& } \\
\text { ACP }\end{array}$} & \multirow[t]{3}{*}{ AA } & Male & $\mathbf{R R}$ & 5 & 32 & $14 \%$ & $7.2 \%$ & & & 1.3 & & \\
\hline & & Female & $\mathbf{R R}$ & 15 & 90 & $14 \%$ & $7.7 \%$ & & & 1.1 & & \\
\hline & & Both & $\mathbf{R} \mathbf{R}$ & 20 & 122 & $14 \%$ & $7.6 \%$ & & & 1.1 & & \\
\hline \multirow{3}{*}{ ACP \& USCF } & \multirow[t]{3}{*}{ AA } & Male & Ctl & 12 & 99 & $11 \%$ & $5.7 \%$ & & & & & \\
\hline & & Female & Ctl & 43 & 286 & $13 \%$ & $7.0 \%$ & & & & & \\
\hline & & Both & CtI & 55 & 385 & $13 \%$ & $6.7 \%$ & & & $\mathrm{~b}$ & & \\
\hline \multirow{6}{*}{ UCSF } & \multirow[t]{3}{*}{ White } & Male & RR & 19 & 190 & $9 \%$ & $4.8 \%$ & & & & & 1.1 \\
\hline & & Female & $\mathbf{R R}$ & 49 & 396 & $11 \%$ & $5.8 \%$ & & & & C & $1.6^{d}$ \\
\hline & & Both & RR & 68 & 586 & $10 \%$ & $5.5 \%$ & & & & & 1.5 \\
\hline & White & Male & Ctl & 19 & 217 & $8 \%$ & $4.2 \%$ & & & & & \\
\hline & & Female & Ctl & 30 & 395 & $7 \%$ & $3.7 \%$ & & & & & d \\
\hline & & Both & CtI & 49 & 612 & $7 \%$ & $3.8 \%$ & & & & & \\
\hline
\end{tabular}

a, $p=0.022 ;{ }^{b}, p=0.058 ;{ }^{c}, p=0.052 ;{ }^{d}, p=0.045 ;{ }^{e}, p=0.065$. Fisher's exact test. Letters are duplicated to facilitate comparisons. ACP, Accelerated Cure Project; RR, relapsing remitting; SP, secondary progressive; MAF, minor allele frequency. 
Table 3 Effect of the STK11 SNP on clinical parameters in African American PwMS

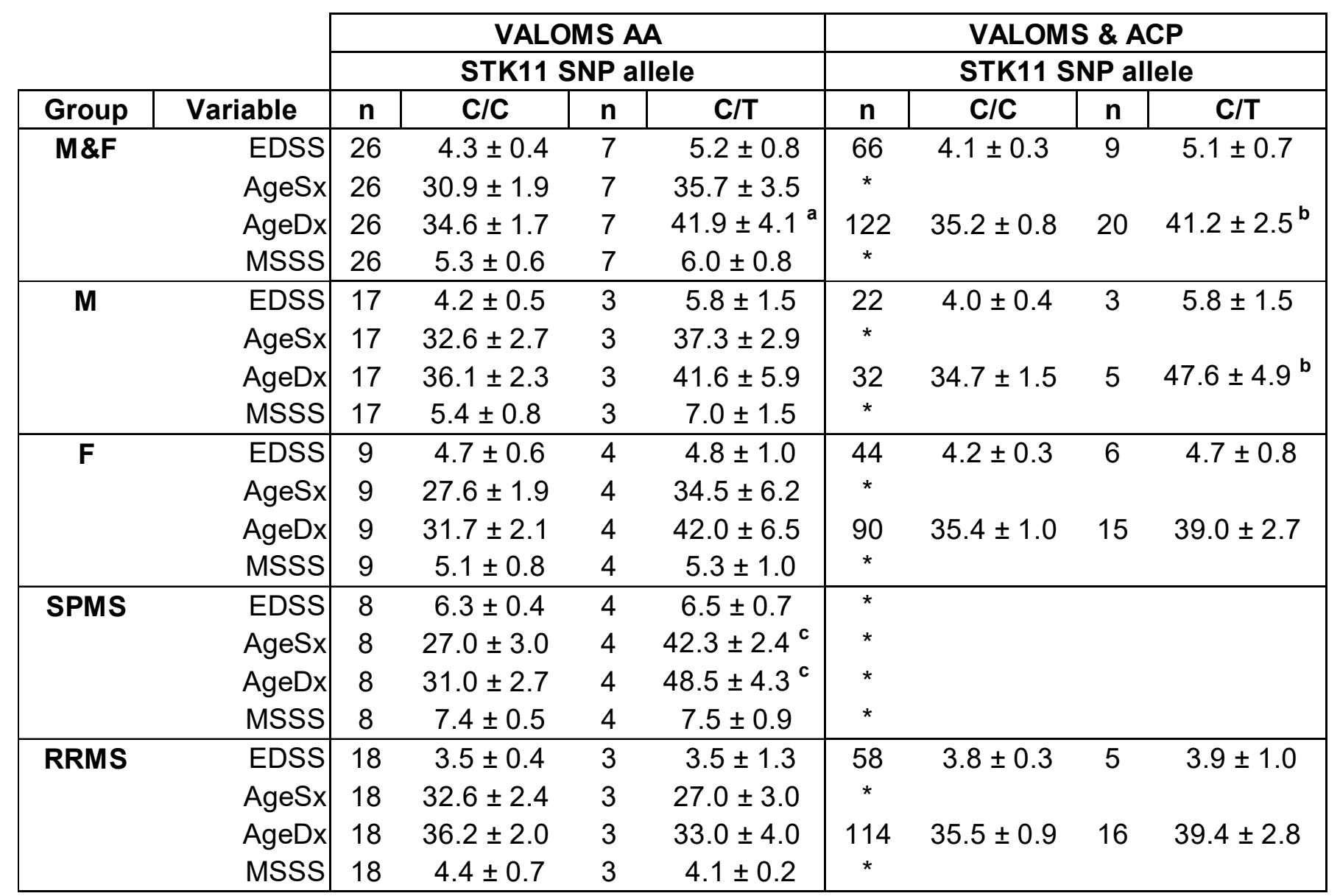

a, $P=0.073 ;{ }^{b}, P<0.05 ;{ }^{c}, P<0.01 .{ }^{*}$, no AgeSx, MSSS, or SPMS in ACP group. Data is mean \pm se. ACP, Accelerated Cure Project, EDSS, expanded disability severity scale; AgeSx, age at symptom onset; AgeDx, age at diagnosis; MSSS, multiple sclerosis severity score; RR, relapsing remitting; SP, secondary progressive 
Table 4 Effect of the STK11 SNP on clinical parameters in White PwMS

\begin{tabular}{|c|c|c|c|c|c|c|c|c|c|}
\hline \multirow[b]{3}{*}{ Group } & \multirow[b]{3}{*}{ Variable } & \multicolumn{4}{|c|}{ VALOMS White } & \multicolumn{4}{|c|}{ UCSF White } \\
\hline & & \multicolumn{4}{|c|}{ STK11 SNP allele } & \multicolumn{4}{|c|}{ STK11 SNP allele } \\
\hline & & $\mathbf{n}$ & $\mathrm{C} / \mathrm{C}$ & $\mathbf{n}$ & $\mathbf{C} / \mathbf{T}$ & $\mathbf{n}$ & $\mathrm{C} / \mathrm{C}$ & $\mathbf{n}$ & $\mathrm{C} / \mathrm{T}$ \\
\hline \multirow[t]{4}{*}{ M\&F } & EDSS & 52 & $4.2 \pm 0.3$ & 5 & $3.9 \pm 1.3$ & 577 & $2.8 \pm 0.1$ & 65 & $2.3 \pm 0.2^{a}$ \\
\hline & AgeSx & 52 & $35.6 \pm 1.6$ & 5 & $33.6 \pm 3.4$ & 581 & $32.8 \pm 0.4$ & 66 & $32.1 \pm 1.1$ \\
\hline & AgeDx & 52 & $42.1 \pm 1.4$ & 5 & $37.4 \pm 2.5$ & & $\mathrm{n} / \mathrm{a}$ & & $\mathrm{n} / \mathrm{a}$ \\
\hline & MSSS & 52 & $4.9 \pm 0.4$ & 5 & $4.3 \pm 0.4$ & 577 & $3.5 \pm 0.1$ & 66 & $3.0 \pm 0.3$ \\
\hline \multirow[t]{4}{*}{$\mathbf{M}$} & EDSS & 40 & $4.3 \pm 0.3$ & 4 & $3.0 \pm 1.2$ & 189 & $3.0 \pm 0.1$ & 19 & $2.1 \pm 0.3^{a}$ \\
\hline & AgeSx & 40 & $37.0 \pm 1.9$ & 4 & $35.0 \pm 4.0$ & 190 & $33.4 \pm 0.7$ & 19 & $29.3 \pm 2.4^{b}$ \\
\hline & AgeDx & 40 & $42.2 \pm 1.7$ & 4 & $39.5 \pm 2.6$ & & $\mathrm{n} / \mathrm{a}$ & & $\mathrm{n} / \mathrm{a}$ \\
\hline & MSSS & 40 & $5.2 \pm 0.4$ & 4 & $3.0 \pm 1.2$ & 189 & $3.6 \pm 0.2$ & 19 & $3.2 \pm 0.6$ \\
\hline \multirow[t]{4}{*}{$\mathbf{F}$} & EDSS & 12 & $3.9 \pm 0.6$ & 1 & 7.5 & 388 & $2.7 \pm 0.1$ & 46 & $2.4 \pm 0.2$ \\
\hline & AgeSx & 12 & $30.8 \pm 3.0$ & 1 & 28 & 391 & $32.5 \pm 0.5$ & 47 & $33.2 \pm 1.2$ \\
\hline & AgeDx & 12 & $41.5 \pm 2.3$ & 1 & 29 & & $\mathrm{n} / \mathrm{a}$ & & $\mathrm{n} / \mathrm{a}$ \\
\hline & MSSS & 12 & $3.8 \pm 0.8$ & 1 & 9.7 & 388 & $3.5 \pm 0.1$ & 47 & $2.9 \pm 0.3$ \\
\hline \multirow[t]{4}{*}{ SPMS } & EDSS & 26 & $5.3 \pm 0.4$ & 1 & 6.5 & & & & \\
\hline & AgeSx & 26 & $37.2 \pm 2.1$ & 1 & 28 & & & & \\
\hline & AgeDx & 26 & $44.5 \pm 1.6$ & 1 & 35 & & & & \\
\hline & MSSS & 26 & $5.8 \pm 0.5$ & 1 & 6.3 & & & & \\
\hline \multirow[t]{4}{*}{ RRMS } & EDSS & 26 & $3.1 \pm 0.3$ & 4 & $3.3 \pm 1.4$ & & & & \\
\hline & AgeSx & 26 & $33.9 \pm 2.4$ & 4 & $35.0 \pm 4.0$ & & & & \\
\hline & AgeDx & 26 & $39.7 \pm 2.2$ & 4 & $38.0 \pm 3.7$ & & & & \\
\hline & MSSS & 26 & $3.9 \pm 0.5$ & 4 & $3.9 \pm 2.0$ & & & & \\
\hline
\end{tabular}

a, $P<0.05 ; ~{ }^{b}, P=0.088$. EDSS, expanded disability severity scale; AgeSx, age at symptom onset; AgeDx, age at diagnosis; MSSS, multiple sclerosis severity score; RR, relapsing remitting; SP, secondary progressive. 


\section{Supplemental Table 1: Demographic and clinical features of PwMS}

\begin{tabular}{|c|c|c|c|c|c|c|c|c|c|c|c|}
\hline Cohort & Race & Gender & MS Type & $\mathbf{N}$ & Age Onset & $\begin{array}{c}\text { Age } \\
\text { Diagnosis }\end{array}$ & Years Dx-Sx & $\begin{array}{c}\text { Duration from } \\
\text { Diagnosis }\end{array}$ & $\begin{array}{c}\text { Duration from } \\
\text { Onset }\end{array}$ & EDSS & MSSS \\
\hline VALOMS & \multirow[t]{9}{*}{ AA } & \multirow[t]{3}{*}{ Male } & RR & 13 & $34.1 \pm 3.0$ & $37.7 \pm 2.4$ & $2.5 \pm 2.0$ & $7.1 \pm 1.6$ & $9.5 \pm 2.2$ & $3.3 \pm 0.5 \mathbf{b}$ & $4.3 \pm 0.8 \mathrm{~b}$ \\
\hline & & & SP & 7 & $31.9 \pm 4.0$ & $35.6 \pm 4.4 \mathrm{a}$ & $3.7 \pm 1.1$ & $8.1 \pm 1.9$ & $11.9 \pm 2.5$ & $6.5 \pm 0.6 \mathbf{f}$ & $8.0 \pm 0.5$ \\
\hline & & & Both & 20 & $34.0 \pm 2.3$ & $37.0 \pm 2.1$ & $3.0 \pm 1.4$ & $7.4 \pm 1.2$ & $10.3 \pm 1.7$ & $4.4 \pm 0.5$ & $5.8 \pm 0.7$ \\
\hline & & \multirow[t]{3}{*}{ Female } & $\mathbf{R R}$ & 8 & $28.1 \pm 2.1$ & $32.6 \pm 2.6$ & $4.5 \pm 2.1$ & $8.5 \pm 1.8$ & $13 \pm 2.7$ & $3.8 \pm 0.7 \mathbf{b}$ & $4.3 \pm 0.8$ \\
\hline & & & SP & 5 & $32.4 \pm 5.3$ & $38.6 \pm 5.6$ & $6.2 \pm 2.1$ & $9.4 \pm 2.4$ & $15.6 \pm 2.4$ & $6.1 \pm 0.2 \mathbf{f}$ & $6.5 \pm 0.4$ \\
\hline & & & Both & 13 & $29.8 \pm 2.3$ & $34.9 \pm 2.7$ & $5.2 \pm 1.5$ & $8.8 \pm 1.4$ & $14 \pm 1.9$ & $4.7 \pm 0.5$ & $5.1 \pm 0.6$ \\
\hline & & \multirow[t]{3}{*}{ Both } & RR & 21 & $31.8 \pm 2.1$ & $35.8 \pm 1.8$ & $3.3 \pm 1.5$ & $7.6 \pm 1.2$ & $10.8 \pm 1.7$ & $3.5 \pm 0.4 \mathbf{b}$ & $4.3 \pm 0.6 \mathbf{b}$ \\
\hline & & & SP & 12 & $32.1 \pm 3.0$ & $36.8 \pm 3.3 \mathrm{a}$ & $4.8 \pm 1.1$ & $8.7 \pm 1.4$ & $13.4 \pm 1.8$ & $6.3 \pm 0.3 \mathbf{c}, \mathbf{f}$ & $7.4 \pm 0.4 \mathrm{c}$ \\
\hline & & & Both & 33 & $32.3 \pm 1.7$ & $36.2 \pm 1.7 \mathrm{a}$ & $3.8 \pm 1.0$ & $7.9 \pm 0.9$ & $11.8 \pm 1.3$ & $4.5 \pm 0.4$ & $5.5 \pm 0.5$ \\
\hline & \multirow[t]{9}{*}{ White } & \multirow[t]{3}{*}{ Male } & RR & 24 & $34.0 \pm 2.4$ & $39.3 \pm 2.3$ & $5.3 \pm 1.8$ & $8.5 \pm 1.4$ & $13.8 \pm 2.2$ & $3.1 \pm 0.3 \mathbf{e}$ & $3.9 \pm 0.5 \mathbf{e}$ \\
\hline & & & SP & 20 & $39.2 \pm 2.3 \mathbf{h}$ & $44.2 \pm 1.9$ & $5.0 \pm 1.0$ & $8.3 \pm 0.9$ & $13.3 \pm 1.3$ & $5.5 \pm 0.4 \mathbf{h}$ & $6.3 \pm 0.5 h$ \\
\hline & & & Both & 44 * & $36.4 \pm 1.7$ & $41.5 \pm 1.6$ & $5.2 \pm 1.1$ & $8.4 \pm 0.8$ & $13.6 \pm 1.3$ & $4.2 \pm 0.3 \mathbf{h}$ & $5.0 \pm 0.4 h$ \\
\hline & & \multirow[t]{3}{*}{ Female } & $\mathbf{R R}$ & 6 & $31.0 \pm 4.1$ & $36.5 \pm 3.1$ & $5.5 \pm 2.5$ & $6.3 \pm 1.6$ & $11.8 \pm 2.2$ & $3.3 \pm 0.9$ & $3.8 \pm 1.4$ \\
\hline & & & SP & 7 & $30.2 \pm 4.0$ & $44.0 \pm 3.1$ & $13.7 \pm 3.6$ & $8.6 \pm 1.8$ & $22.3 \pm 4.2 \mathbf{g}$ & $4.9 \pm 0.8 \mathbf{h}$ & $4.5 \pm 1.3 h$ \\
\hline & & & Both & 13 & $30.6 \pm 2.7$ & $40.5 \pm 2.4$ & $9.9 \pm 2.5$ & $7.5 \pm 1.2$ & $17.5 \pm 2.8$ & $4.2 \pm 0.6 \mathbf{h}$ & $4.2 \pm 0.9$ \\
\hline & & \multirow[t]{3}{*}{ Both } & RR & 30 & $33.4 \pm 2.1$ & $38.7 \pm 2.0 \mathrm{~d}$ & $5.3 \pm 1.5$ & $8.1 \pm 1.1$ & $13.4 \pm 1.8$ & $3.1 \pm 0.3 \mathrm{~d}$ & $3.9 \pm 0.5 \mathrm{~d}$ \\
\hline & & & SP & 27 & $36.9 \pm 2.1 \mathrm{~h}$ & $44.1 \pm 1.6$ & $7.3 \pm 1.4$ & $8.3 \pm 0.8$ & $15.6 \pm 1.6$ & $5.3 \pm 0.3 \mathbf{h}$ & $5.8 \pm 0.5 h$ \\
\hline & & & Both & 57 & $35.1 \pm 1.5$ & $41.2 \pm 1.3$ & $6.2 \pm 1.0$ & $8.2 \pm 0.7$ & $14.5 \pm 1.2$ & $4.2 \pm 0.3 \mathbf{h}$ & $4.8 \pm 0.4 \mathbf{h}$ \\
\hline \multirow[t]{3}{*}{ ACP } & \multirow[t]{3}{*}{ AA } & Male & RR & 17 & & $35.9 \pm 2.6$ & & $4.7 \pm 1.0$ & & $3.3 \pm 0.8(5)$ & \\
\hline & & Female & RR & 92 & & $36.1 \pm 1.0$ & & $6.4 \pm 0.6$ & & $4.1 \pm 0.4(37)$ & \\
\hline & & Both & RR & 109 & & $36.1 \pm 0.9$ & & $6.2 \pm 0.6$ & & $4.0 \pm 0.4(42)$ & \\
\hline \multirow[t]{3}{*}{ UCSF } & \multirow[t]{3}{*}{ White } & Male & RR & 209 & $32.9 \pm 0.6(208)$ & & & & $13.0 \pm 0.7(208)$ & $2.9 \pm 0.1(208)$ & $3.6 \pm 0.2(208)$ \\
\hline & & Female & RR & 445 & $32.6 \pm 0.4(437)$ & & & & $12.2 \pm 0.5(437)$ & $2.7 \pm 0.1(434)$ & $3.4 \pm 0.1(435)$ \\
\hline & & Both & $\mathbf{R R}$ & 654 & $32.7 \pm 0.4(645)$ & & & & $12.5 \pm 0.4(645)$ & $2.7 \pm 0.1(642)$ & $3.5 \pm 0.1(643)$ \\
\hline
\end{tabular}

*, Demographic data available for 44 of the 45 male White patients.

a, $\mathrm{P}<0.05$ versus corresponding VA White patients; $b, \mathrm{P}<0.05$ versus corresponding VA AA SPMS patients; $c, P<0.05$ versus all VA White SPMS patients; $d, P<0.05$ versus corresponding VA White SPMS patients; $e, P<0.05$ versus corresponding White male SPMS patients; $f$, $P<0.05$ versus corresponding ACP patients; $g, P<0.05$ versus White female patients; $h, P<0.05$ versus corresponding White patients. Data is mean \pm se. $N$ is number in all groups unless otherwise indicated in parentheses. ACP, Accelerated Cure Project, Dx, age at diagnosis; Sx, age at first symptom onset; EDSS, expanded disability severity scale; MSSS, multiple sclerosis severity score; RR, relapsing remitting; SP, secondary progressive. 


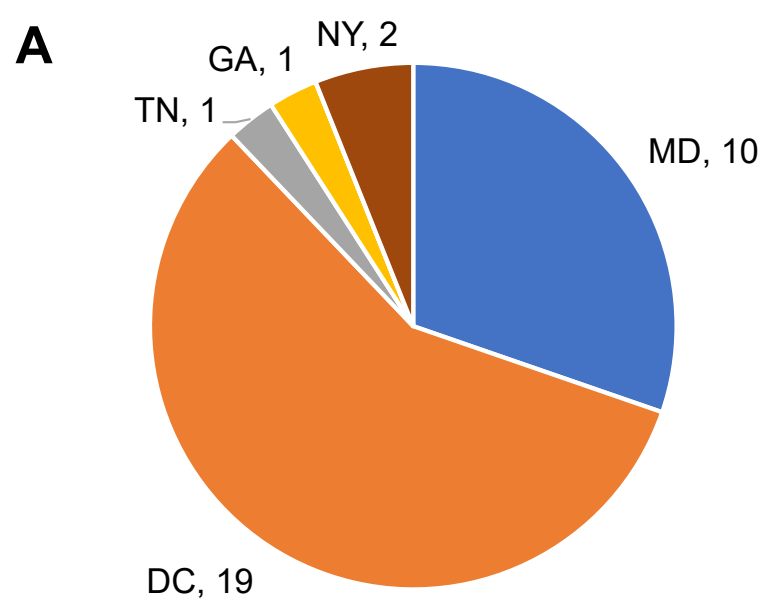

VALOMS, AA (33)
B

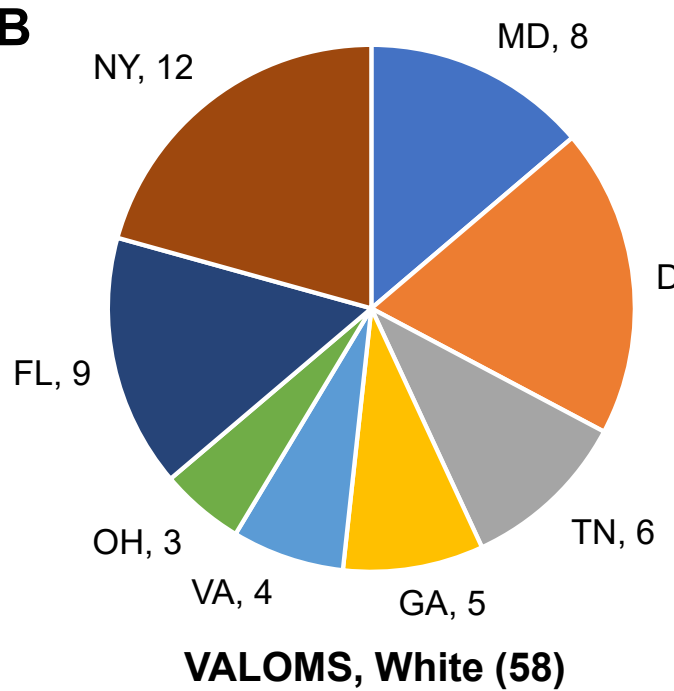

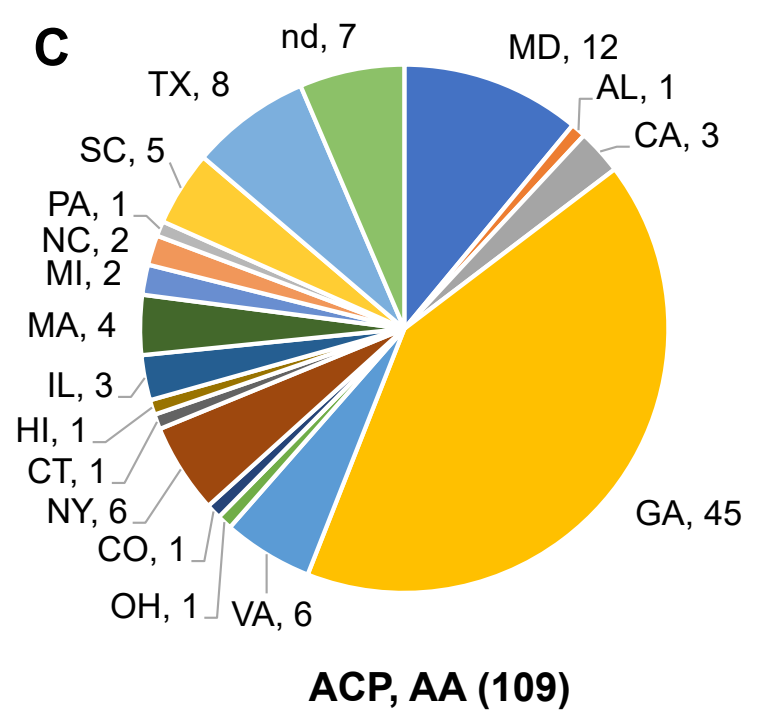

Figure 1 


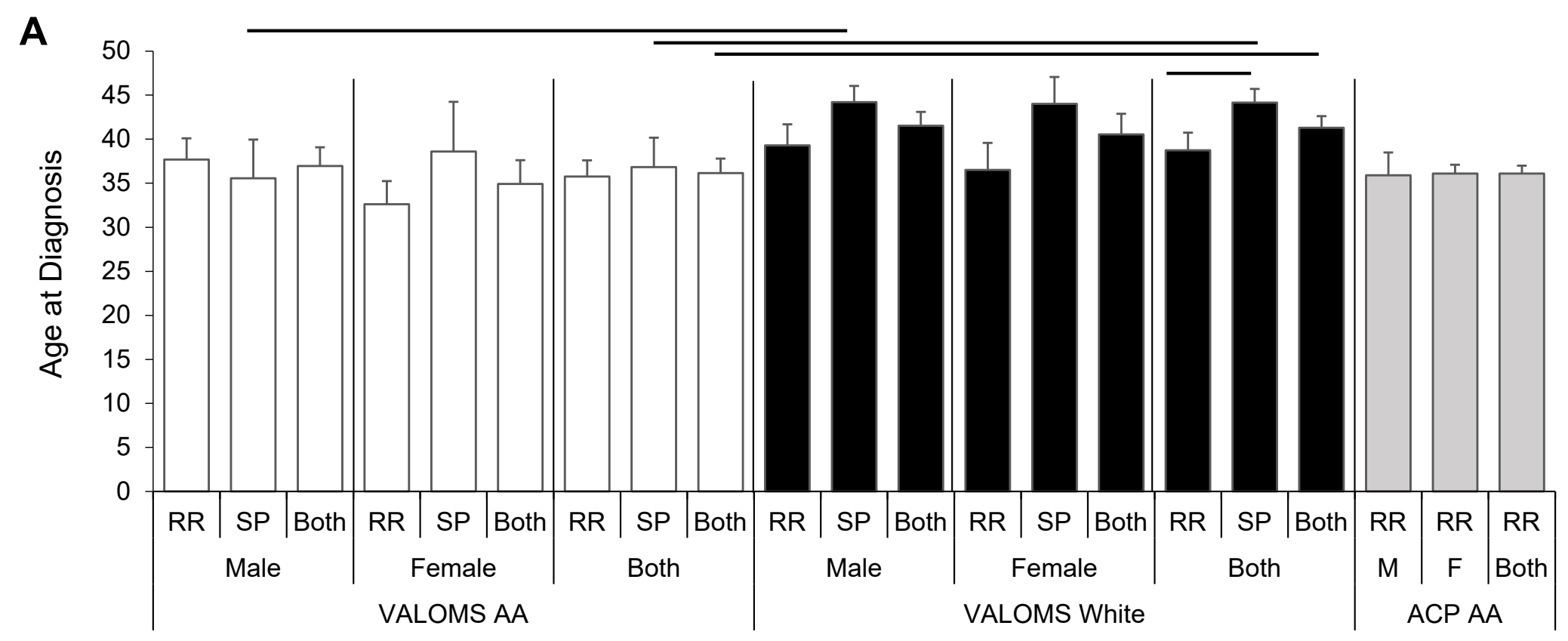

Figure 2A 


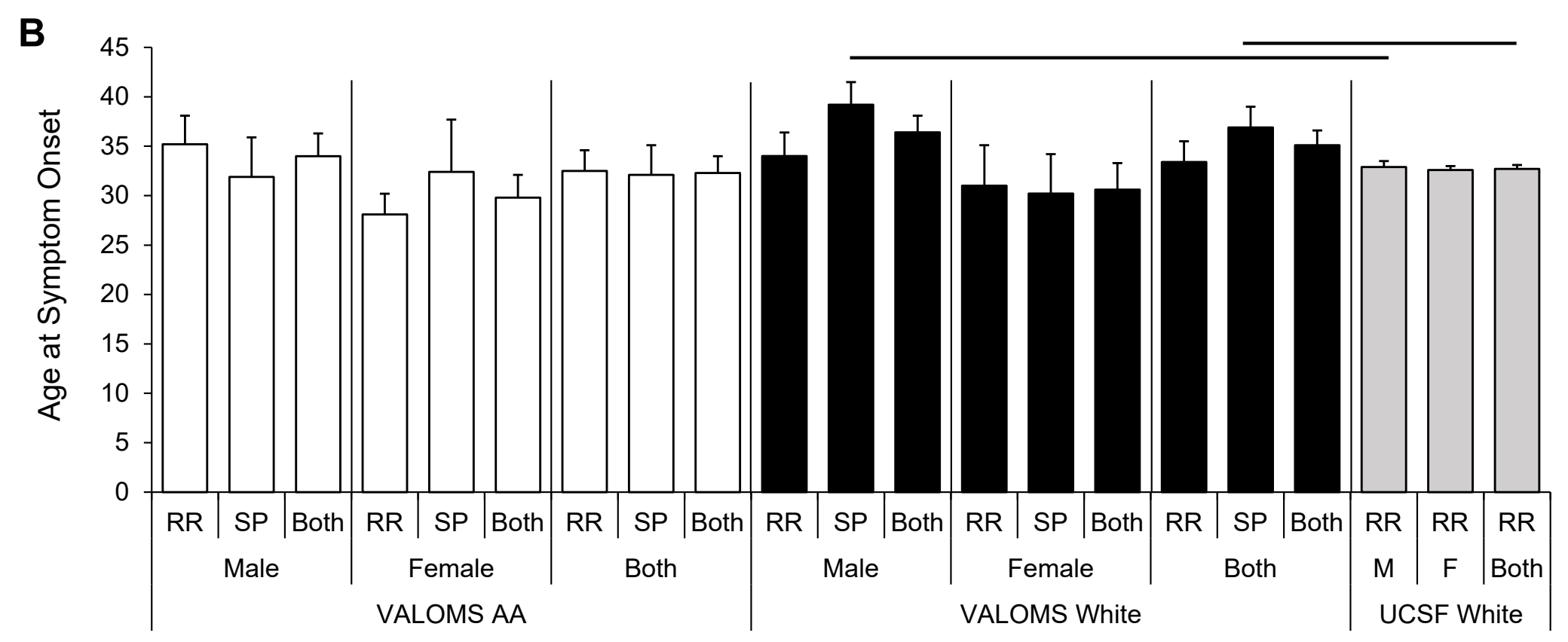

Figure 2B 


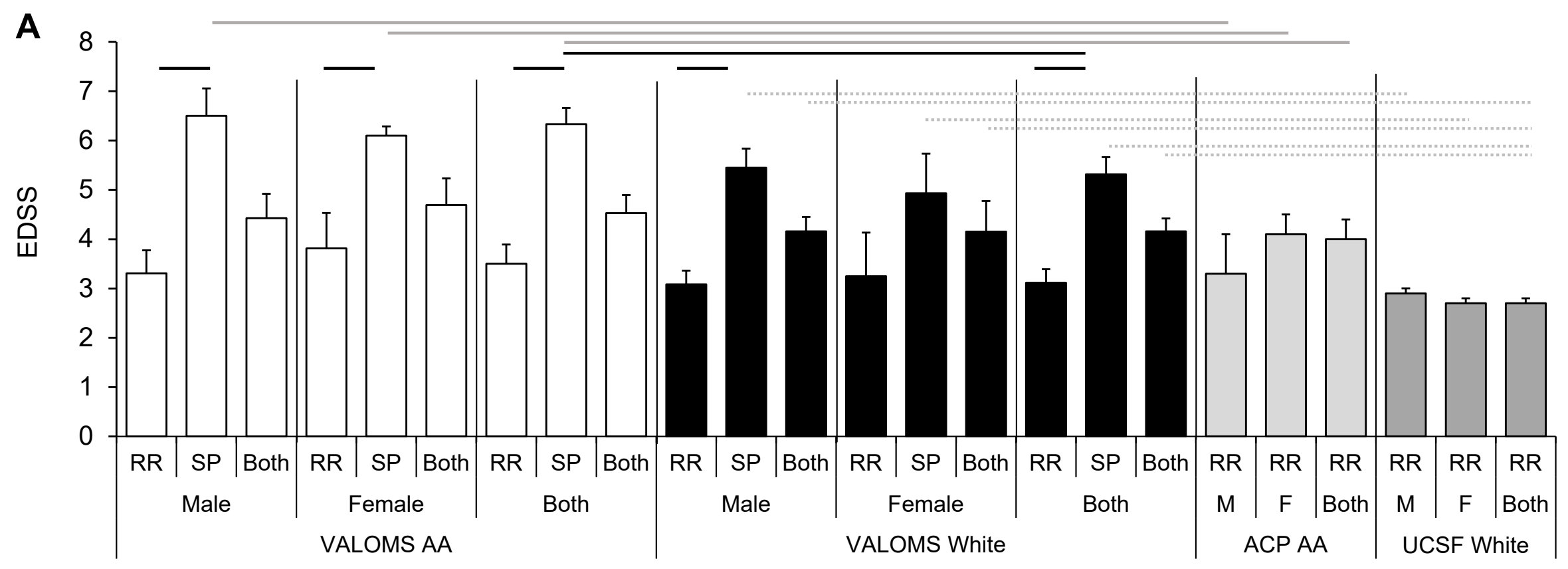

Figure 3A 


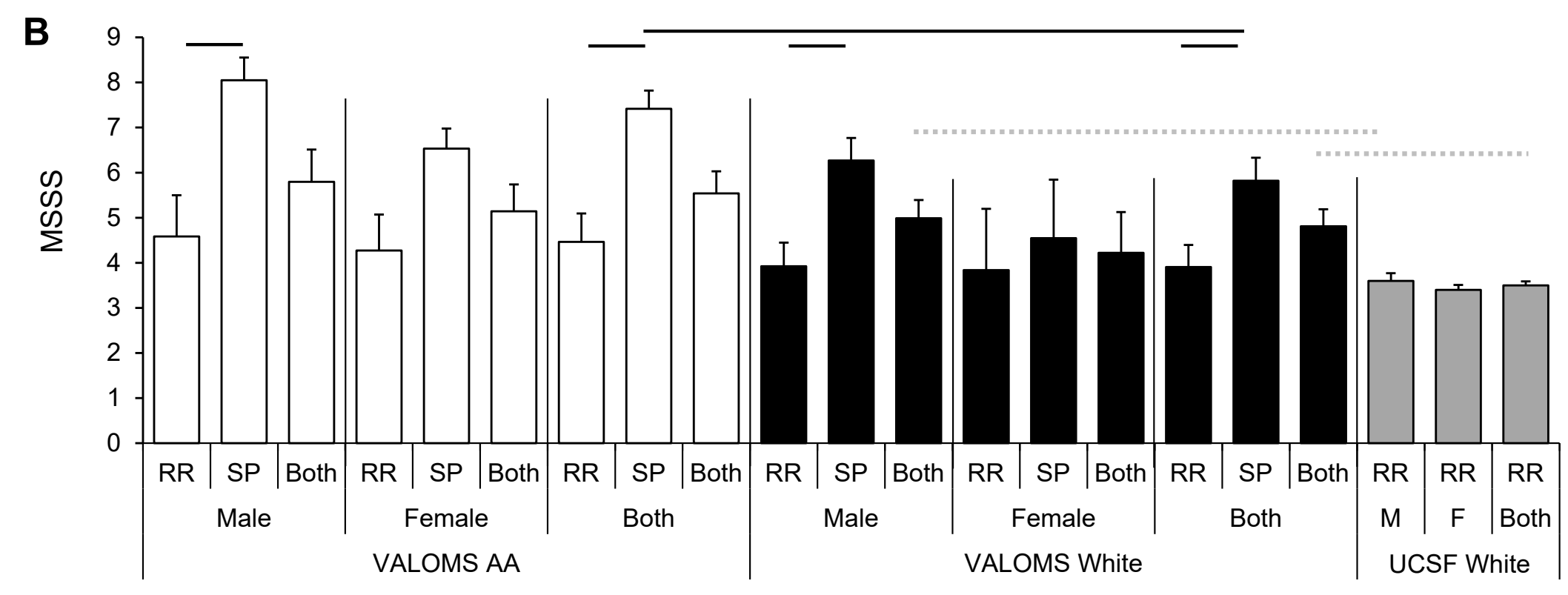

Figure 3B 
0.058

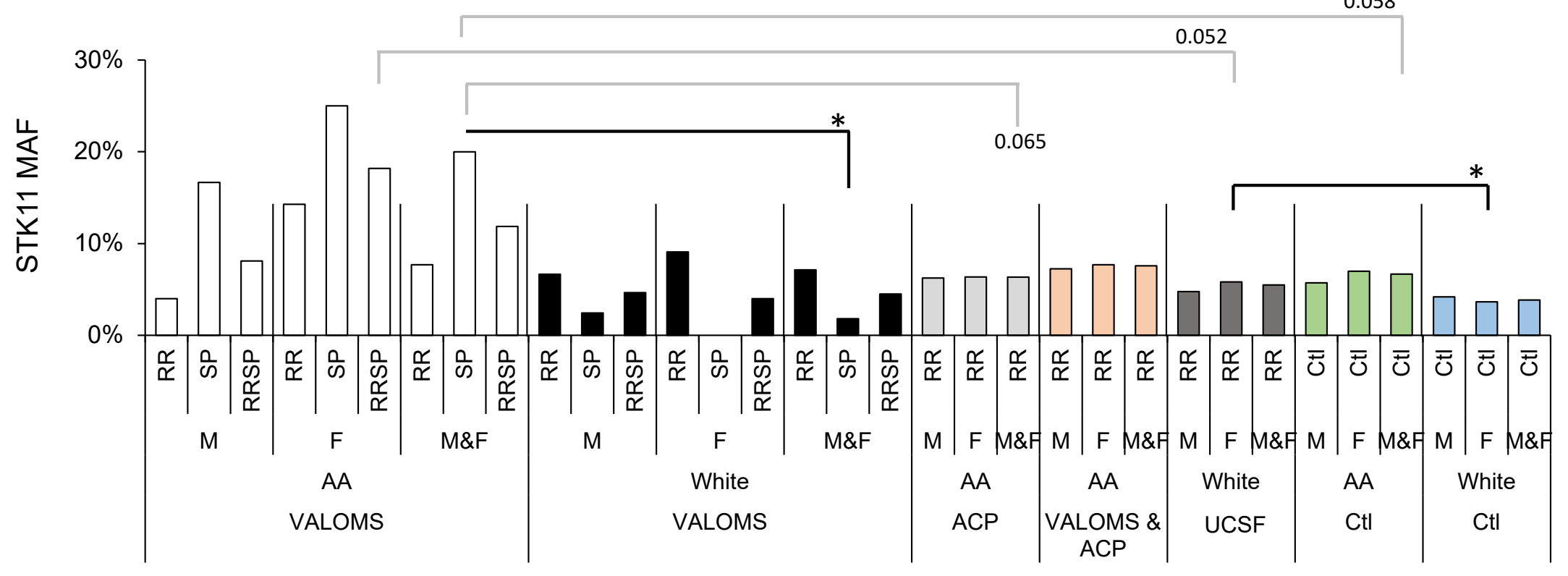

Figure 4 
A

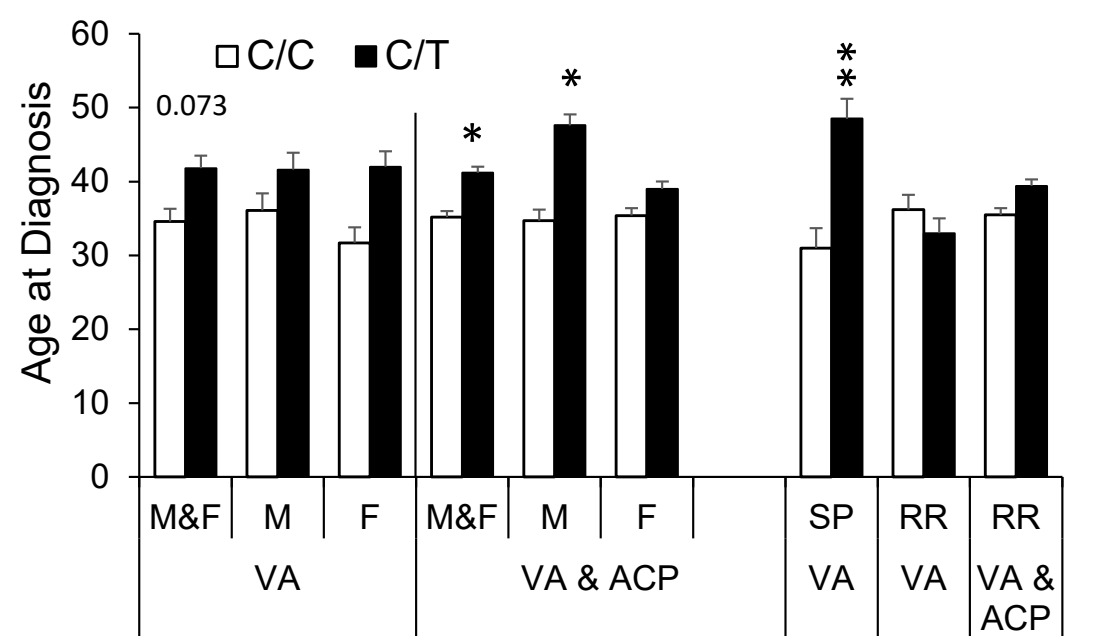

B

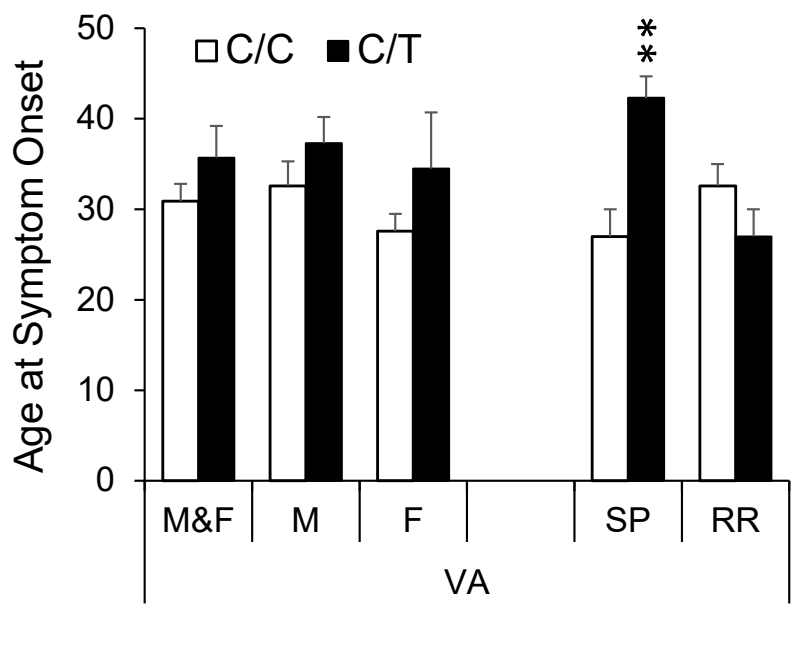

Figure 5 
Figures

A

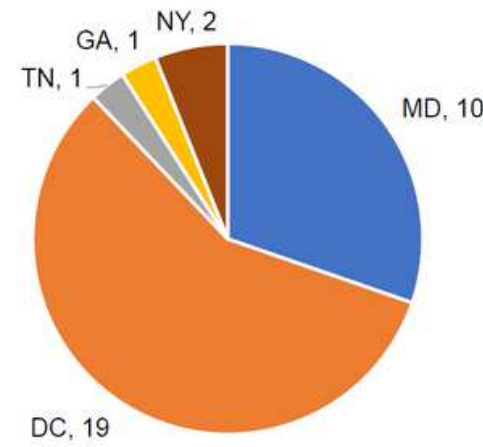

VALOMS, AA (33)
B

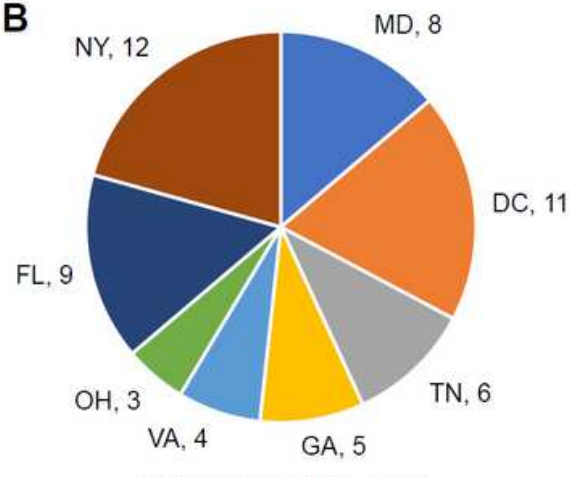

VALOMS, White (58)

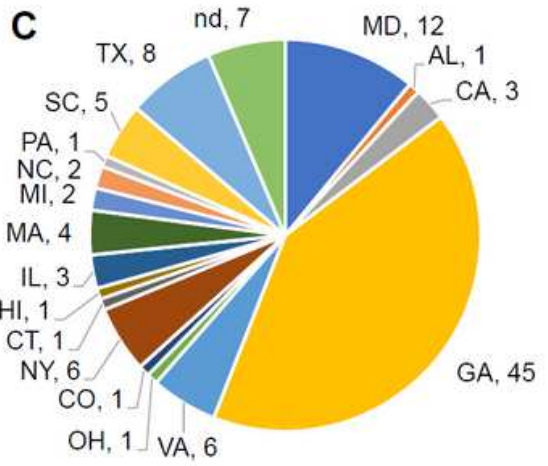

ACP, AA (109)

\section{Figure 1}

Regional distribution of VALOMS and ACP patients with MS Values for the number of male and female patients are shown by state for VALOMS (A) AA and (B) White PwMS; and (C) AA PwMS from ACP. nd, no data. 

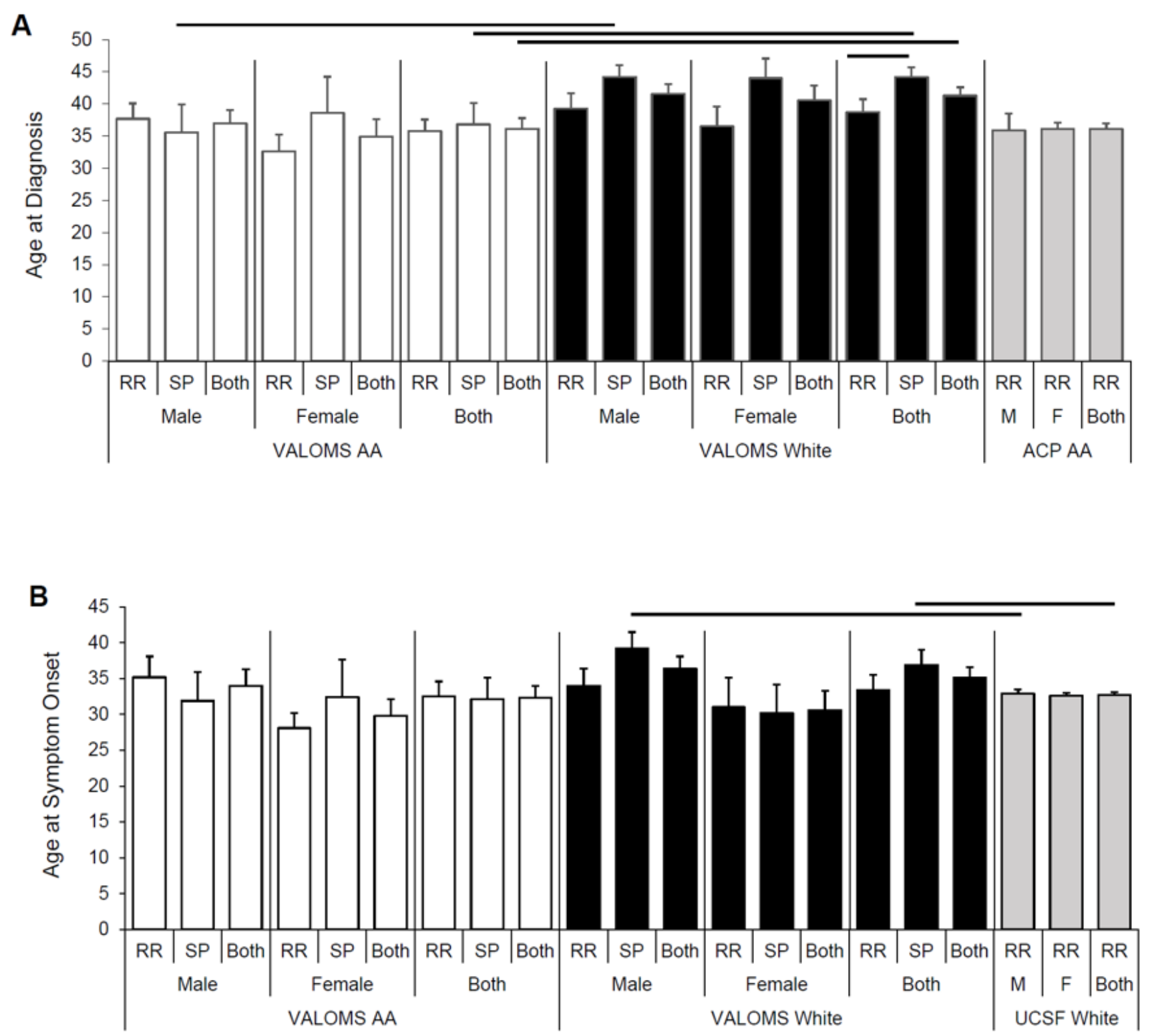

Figure 2

Age at diagnosis of VALOMS AA and White patients with MS Average age at (A) diagnosis and (B) symptom onset for VALOMS AA (white bars), VALOMS White (black bars), ACP AA (gray bars in A), and UCSF White (gray bars in $B)$ PwMS. Black lines indicate significance between groups $(P<0.05)$. Data are mean \pm se and are taken from Table 1 . 

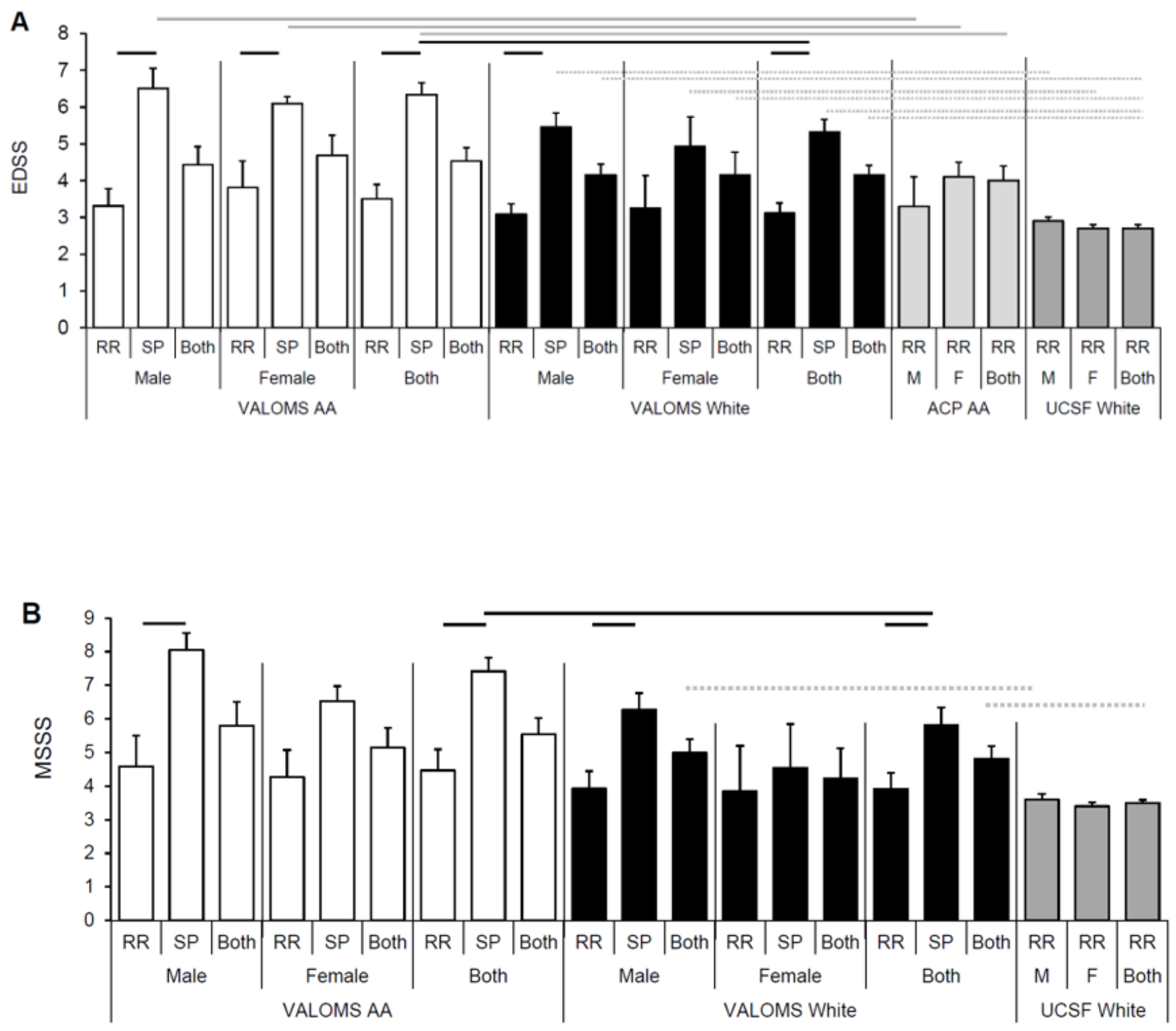

Figure 3

MS disease severity in AA and White patients with MS Average (A) EDSS scores measured at last exam and (B) MSSS for VALOMS AA (white bars), VALOMS White (black bars), ACP AA (light gray bars in A), and UCSF White (dark gray bars in A and B) PwMS. Significance $(P<0.05)$ is indicated by black lines comparing VALOMS AA and White patients; gray lines comparing VALOMS to ACP AA PWMS; and dashed lines comparing VALOMS to UCSF White PWMS. Data is mean \pm se and are taken from Table 1. 


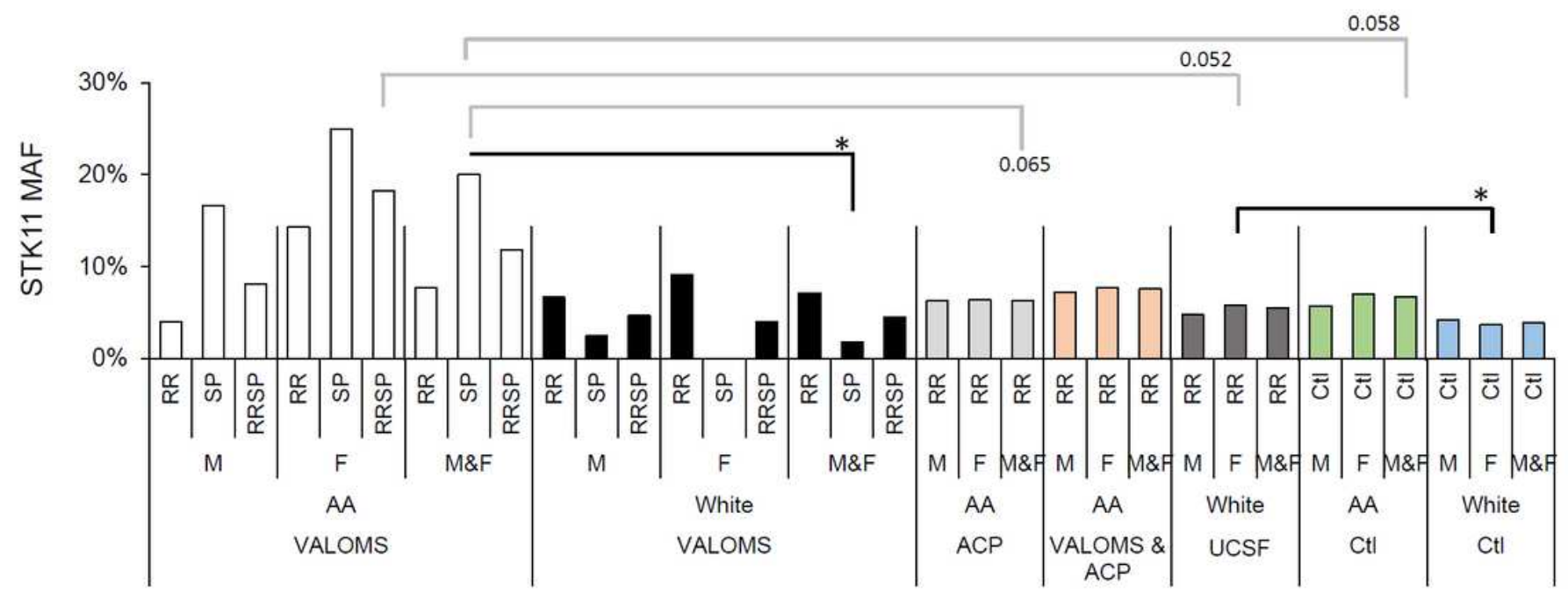

Figure 4

STK11 SNP prevalence The minor allele frequency (MAF) for the $\mathrm{C} / \mathrm{T}$ genotype is shown for all groups. Significance $\left({ }^{*}, \mathrm{P}<0.05\right)$ is indicated by black lines; near significance is indicated by gray lines. Data are taken from Table 2.

A

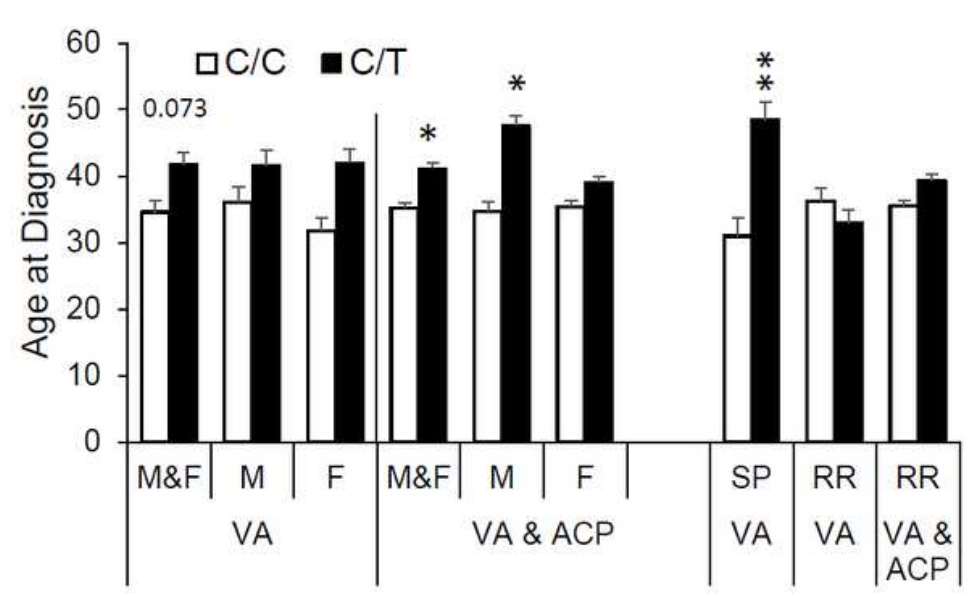

B

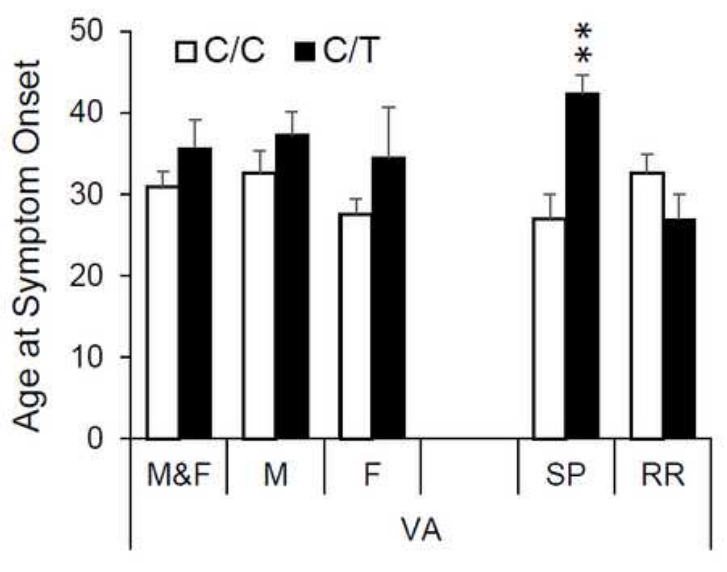

Figure 5

Effect of STK11 SNP on age at diagnosis and age at symptom onset Average (A) age at diagnosis and (B) age at symptom onset for VALOMS AA PWMS and for the combined VALOMS \& ACP AA PWMS (VA \& $A C P$ ), having the $C / C$ (white bars) or $C / T$ (black bars) allele. ${ }^{*}, P<0.05$; $* \star, P<0.005$ versus $C / C$. Data is mean \pm se and are taken from Table 3 . 